\title{
ON BOUNDARY EXTENSION OF MAPPINGS IN METRIC SPACES IN THE TERMS OF PRIME ENDS
}

\author{
Evgeny Sevost'yanov \\ Zhytomyr Ivan Franko State University \\ 40 Bol'shaya Berdichevskaya Str., 10008 Zhytomyr, Ukraine; esevostyanov2009@gmail.com
}

\begin{abstract}
We study the boundary behavior of the so-called ring $Q$-mappings obtained as a natural generalization of mappings with bounded distortion. We establish a series of conditions imposed on a function $Q(x)$ for the continuous extension of given mappings with respect to prime ends in domains with regular boundaries in metric spaces.
\end{abstract}

\section{Introduction}

Problems of continuous extension of mappings with finite distortion in terms of prime ends in $\mathbf{R}^{n}$ were recently investigated in [GRY] for $n=2$, and in $[\mathrm{KR}]$ for $n \geqslant 2$. The latter paper was devoted to the case of homeomorphisms between spatial domains with regular prime ends. However, the case of mappings with branching was not considered in these papers. The present paper solves similar problems in general metric spaces and not only for homeomorphisms but also for more general open discrete mappings, cf. [ABBS], [A] and $\left[\mathrm{Sev}_{1}\right]$.

The following definitions are from $[\mathrm{A}]$ and $[\mathrm{ABBS}]$. Given a metric space $(X, d, \mu)$ with a measure $\mu$, a domain in $X$ is an open path-connected set in $X$. Recall that $X$ is locally (path) connected if every neighborhood of a point $x \in X$ contains a (path) connected neighborhood. We define the Mazurkiewicz distance $d_{M}$ on $X$ by $d_{M}(x, y)=\inf \operatorname{diam} E$, where the infimum is over all connected sets $E \subset X$ containing $x, y \in E$. Clearly, $d_{M}$ is a metric on $X$. Let $\gamma$ be a curve in $\Omega$. We define its diameter as follows:

$$
\operatorname{diam} \gamma:=\sup d(x, y)
$$

where the supremum is taken over all points $x, y \in \gamma$. When $x, y \in X$, we have

$$
d_{M}(x, y) \geqslant d(x, y)
$$

Set

$$
B\left(x_{0}, r\right):=\left\{x \in X: d\left(x, x_{0}\right)<r\right\}, \quad S\left(x_{0}, r\right):=\left\{x \in X: d\left(x, x_{0}\right)=r\right\} .
$$

From now on we assume that the space $X$ is complete and supports a $p$-Poincare inequality, and that the measure is doubling (see [ABBS]). In this case, a space $X$ is locally connected (see [ABBS, Section 2]), and proper (see [BB, Proposition 3.1]). The relation between spaces supporting the $p$-Poincaré inequality and the local connectedness is due to Semmes [Se], cf. the discussion at the end of Section 2 in [ABBS].

https://doi.org/10.5186/aasfm.2019.4405

2010 Mathematics Subject Classification: Primary 30L10; Secondary 30C65.

Key words: Mappings with finite and bounded distortion, prime ends, metric spaces, boundary behavior. 
If $X$ is also connected then there exist constants $C>0$ and $q>0$ such that for all $x \in X, 0<r \leqslant R$ and $y \in B(x, R)$,

$$
\frac{\mu(B(y, r))}{\mu(B(x, R))} \leqslant C\left(\frac{r}{R}\right)^{q}
$$

see [ABBS, (2.2)]. Let $\Omega \varsubsetneqq X$ be a bounded domain in $X$, i.e. a bounded nonempty connected open subset of $X$ that is not the whole space $X$ itself. The completion of the metric space $\left(\Omega, d_{M}\right)$ is denoted $\bar{\Omega}^{M}$, and $d_{M}$ extends in the standard way to $\bar{\Omega}^{M}$ : For $d_{M}$-Cauchy sequences $\left\{x_{n}\right\}_{n=1}^{\infty},\left\{y_{n}\right\}_{n=1}^{\infty} \in \Omega$ we define the equivalence relation

$$
\left\{x_{n}\right\}_{n=1}^{\infty} \sim\left\{y_{n}\right\}_{n=1}^{\infty} \text { if } \lim _{n \rightarrow \infty} d_{M}\left(x_{n}, y_{n}\right)=0 .
$$

Note that every Cauchy sequence is trivially equivalent to any of its subsequences.

The collection of all equivalence classes of $d_{M}$-Cauchy sequences can be formally considered to be $\bar{\Omega}^{M}$, but we will identify equivalence classes of $d_{M^{-}}$Cauchy sequences having a limit in $\Omega$ with that limit point. By considering equivalence classes of $d_{M^{-}}$ Cauchy sequences without limits in $\Omega$ we define the boundary of $\Omega$ with respect to $d_{M}$ as $\partial_{M} \Omega=\bar{\Omega}^{M} \backslash \Omega$. Since $X$ is proper, we know that $\Omega$ is locally compact with respect to $d_{M}$, and it follows that $\Omega$ is an open subset of $\bar{\Omega}^{M}$. We extend the original metric $d_{M}$ on $\Omega$ to $\bar{\Omega}^{M}$ by setting

$$
d_{M}\left(x^{*}, y^{*}\right)=\lim _{n \rightarrow \infty} d_{M}\left(x_{n}, y_{n}\right)
$$

if $x^{*}=\left\{x_{n}\right\}_{n=1}^{\infty} \in \bar{\Omega}^{M}$ and $y^{*}=\left\{y_{n}\right\}_{n=1}^{\infty} \in \bar{\Omega}^{M}$. This is well defined and an extension of $d_{M}$.

We call a bounded connected set $E \varsubsetneqq \Omega$ an acceptable set if $\bar{E} \cap \partial \Omega \neq \varnothing$. By discussion in [ABBS], we know that boundedness and connectedness of an acceptable set $E$ implies that $\bar{E}$ is compact and connected. Furthermore, $E$ is infinite, as otherwise we would have $\bar{E}=E \subset \Omega$. Therefore, $\bar{E}$ is a continuum. Recall that a continuum is a connected compact set containing at least two points.

We call a sequence $\left\{E_{k}\right\}_{k=1}^{\infty}$ of acceptable sets a chain if it satisfies the following conditions:

1. $E_{k+1} \subset E_{k}$ for all $k=1,2, \ldots$,

2. dist $\left(\Omega \cap \partial E_{k+1}, \Omega \cap \partial E_{k}\right)>0$ for all $k=1,2, \ldots$,

3. The impression $\bigcap_{k=1}^{\infty} \overline{E_{k}} \subset \partial \Omega$.

Here we have used a notation $\operatorname{dist}(A, B):=\inf _{x \in A, y \in B} d(x, y)$, where $d$ is a metric in a given metric space. The definition of chain given above corresponds to work [ABBS], cf. [A] and [ES].

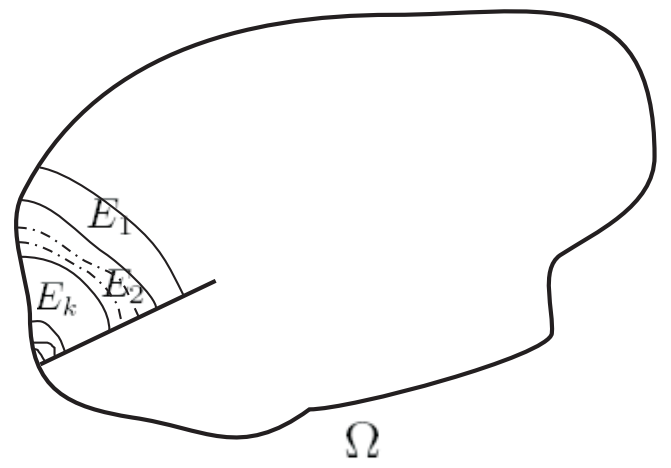

Figure 1. A prime end in domain $\Omega$. 
We say that a chain $\left\{E_{k}\right\}_{k=1}^{\infty}$ divides the chain $\left\{F_{k}\right\}_{k=1}^{\infty}$ if for each $k$ there exists $l_{k}$ such that $E_{l_{k}} \subset F_{k}$. Two chains are equivalent if they divide each other. A collection of all mutually equivalent chains is called an end and denoted $\left[E_{k}\right]$, where $\left\{E_{k}\right\}_{k=1}^{\infty}$ is any of the chains in the equivalence class. The impression of $\left[E_{k}\right]$, denoted $I\left[E_{k}\right]$, is defined as the impression of any representative chain. The collection of all ends is called the end boundary and is denoted $\partial_{E} \Omega$. We say that an end $\left[E_{k}\right]$ is a prime end if it is not divisible by any other end. The collection of all prime ends is called the prime end boundary and is denoted $E_{\Omega}$.

We say that a sequence of points $\left\{x_{n}\right\}_{n=1}^{\infty}$ in $\Omega$ converges to the end $\left[E_{k}\right]$, and write $x_{n} \rightarrow\left[E_{k}\right]$ as $n \rightarrow \infty$, if for all $k$ there exists $n_{k}$ such that $x_{n} \in E_{k}$ whenever $n \geqslant n_{k}$. If $x_{n} \rightarrow\left[E_{k}\right]$ as $n \rightarrow \infty$, and $\left[E_{k}\right]$ divides $\left[F_{k}\right]$, then $x_{n}$ also converges to $\left[F_{k}\right]$. Convergence of points and ends defines a topology on $\Omega \cup \partial_{E} \Omega$ (see e.g. [ABBS, Proposition 8.4]). In this topology, a collection $C \subset \Omega \cup \partial_{E} \Omega$ of points and ends is closed if whenever (a point or an end) $y \in \Omega \cup \partial_{E} \Omega$ is a limit of a sequence in $C$, then $y \in C$.

In what follows, we set $\bar{\Omega}^{P}:=\Omega \cup E_{\Omega}$. We say that $\Omega$ is finitely connected at a point $x_{0} \in \partial \Omega$ if for every $r>0$ there is an open set $G$ (open in $X$ ) such that $x_{0} \in G \subset B\left(x_{0}, r\right)$ and $G \cap \Omega$ has only finitely many components. If $\Omega$ is finitely connected at every boundary point, then it is called finitely connected at the boundary. The following results have been proved in [ABBS].

Proposition 1.1. Assume that $\Omega$ is finitely connected at the boundary. Then all prime ends have singleton impressions, and every $x \in \partial \Omega$ is the impression of a prime end and is accessible (see [ABBS, Theorem 10.8]).

Proposition 1.2. Assume that $\Omega$ is finitely connected at the boundary. Then there is a homeomorphism $\Phi: \bar{\Omega}^{P} \rightarrow \bar{\Omega}^{M}$ such that $\left.\Phi\right|_{\Omega}$ is the identity map. Moreover, the prime end closure $\bar{\Omega}^{P}$ is metrizable with the metric $m_{P}(x, y):=d_{M}(\Phi(x), \Phi(y))$. The topology on $\bar{\Omega}^{P}$ given by this metric is equivalent to the topology given by the sequential convergence discussed above (see [ABBS, Corollary 10.9]).

Recall, for a given continuous path $\gamma:[a, b] \rightarrow X$ in a metric space $(X, d)$, that its length is the supremum of the sums

$$
\sum_{i=1}^{k} d\left(\gamma\left(t_{i}\right), \gamma\left(t_{i-1}\right)\right)
$$

over all partitions $a=t_{0} \leqslant t_{1} \leqslant \ldots \leqslant t_{k}=b$ of the interval $[a, b]$. The path $\gamma$ is called rectifiable if its length is finite.

Given a family of paths $\Gamma$ in $X$, a Borel function $\varrho: X \rightarrow[0, \infty]$ is called admissible for $\Gamma$, abbr. $\varrho \in \operatorname{adm} \Gamma$, if

$$
\int_{\gamma} \varrho d s \geqslant 1
$$

for all (locally rectifiable) $\gamma \in \Gamma$.

Everywhere further, for any sets $E, F$, and $G$ in $X$, we denote by $\Gamma(E, F, G)$ the family of all continuous curves $\gamma:[0,1] \rightarrow X$ such that $\gamma(0) \in E, \gamma(1) \in F$, and $\gamma(t) \in G$ for all $t \in(0,1)$. Everywhere further $(X, d, \mu)$ and $\left(X^{\prime}, d^{\prime}, \mu^{\prime}\right)$ are metric spaces with metrics $d$ and $d^{\prime}$ and locally finite Borel measures $\mu$ and $\mu^{\prime}$, correspondingly. We will assume that $\mu$ is a Borel measure such that $0<\mu(B)<\infty$ for all balls $B$ in $X$. 
Given $p \geqslant 1$, the $p$-modulus of the family $\Gamma$ is the number

$$
M_{p}(\Gamma)=\inf _{\varrho \in \operatorname{adm} \Gamma} \int_{X} \varrho^{p}(x) d \mu(x) .
$$

Should adm $\Gamma$ be empty, we set $M_{p}(\Gamma)=\infty$. A family of paths $\Gamma_{1}$ in $X$ is said to be minorized by a family of paths $\Gamma_{2}$ in $X$, abbr. $\Gamma_{1}>\Gamma_{2}$, if, for every path $\gamma_{1} \in \Gamma_{1}$, there is a path $\gamma_{2} \in \Gamma_{1}$ such that $\gamma_{2}$ is a restriction of $\gamma_{1}$. In this case,

$$
\Gamma_{1}>\Gamma_{2} \Rightarrow M_{p}\left(\Gamma_{1}\right) \leq M_{p}\left(\Gamma_{2}\right)
$$

(see [Fu, Theorem 1]).

Let $G$ and $G^{\prime}$ be domains with finite Hausdorff dimensions $\alpha$ and $\alpha^{\prime} \geqslant 1$ in spaces $(X, d, \mu)$ and $\left(X^{\prime}, d^{\prime}, \mu^{\prime}\right)$, and let $Q: G \rightarrow[0, \infty]$ be a measurable function. Given $x_{0} \in \partial G$, denote $S_{i}:=S\left(x_{0}, r_{i}\right), i=1,2$, where $0<r_{1}<r_{2}<\infty$. We say that a mapping $f: G \rightarrow G^{\prime}$ is a ring $Q$-mapping at a point $x_{0} \in \partial G$, if the inequality

$$
M_{\alpha^{\prime}}\left(f\left(\Gamma\left(S_{1}, S_{2}, A\right)\right)\right) \leqslant \int_{A \cap G} Q(x) \eta^{\alpha}\left(d\left(x, x_{0}\right)\right) d \mu(x)
$$

holds for any ring

$$
A=A\left(x_{0}, r_{1}, r_{2}\right)=\left\{x \in X: r_{1}<d\left(x, x_{0}\right)<r_{2}\right\}, \quad 0<r_{1}<r_{2}<\infty,
$$

and any measurable function $\eta:\left(r_{1}, r_{2}\right) \rightarrow[0, \infty]$ such that

$$
\int_{r_{1}}^{r_{2}} \eta(r) d r \geqslant 1
$$

holds. We also consider the definition (1.3) for maps $f: G \rightarrow X^{\prime}$, where $G \subset X$ is a domain of Hausdorff dimension $\alpha$, and $X^{\prime}$ is a metric space of Hausdorff dimension $\alpha^{\prime}$.

Remark 1.1. Sometimes, some another (similar) definition of ring $Q$-maps is considered. Let $G$ and $G^{\prime}$ be domains with finite Hausdorff dimensions $\alpha$ and $\alpha^{\prime} \geqslant 1$ in spaces $(X, d, \mu)$ and $\left(X^{\prime}, d^{\prime}, \mu^{\prime}\right)$, and let $Q: G \rightarrow[0, \infty]$ be a measurable function. Following [Sm], we say that a mapping $f: G \rightarrow G^{\prime}$ is a ring $Q$-mapping at a point $x_{0} \in \bar{G}$, if the inequality

$$
M_{\alpha^{\prime}}\left(f\left(\Gamma\left(C_{1}, C_{0}, G\right)\right)\right) \leqslant \int_{A \cap G} Q(x) \eta^{\alpha}\left(d\left(x, x_{0}\right)\right) d \mu(x)
$$

holds for any ring

$$
A=A\left(x_{0}, r_{1}, r_{2}\right)=\left\{x \in X: r_{1}<d\left(x, x_{0}\right)<r_{2}\right\}, \quad 0<r_{1}<r_{2}<\infty,
$$

and any two continua $C_{0} \subset \overline{B\left(x_{0}, r_{1}\right)} \cap G, C_{1} \subset G \backslash B\left(x_{0}, r_{2}\right)$, and any measurable function $\eta:\left(r_{1}, r_{2}\right) \rightarrow[0, \infty]$ such that

$$
\int_{r_{1}}^{r_{2}} \eta(r) d r \geqslant 1
$$

holds.

Observe that (1.3) implies (1.5). In fact, assume that (1.3) holds. Let $C_{0} \subset$ $\overline{B\left(x_{0}, r_{1}\right)} \cap G, C_{1} \subset G \backslash B\left(x_{0}, r_{2}\right)$ be two continua. Assume that $\gamma \in \Gamma\left(C_{1}, C_{0}, G\right)$. Given a curve $\gamma:[0,1] \rightarrow G$, we set $|\gamma|:=\{x \in G: \exists t \in[0,1]: \gamma(t)=x\}$. Note that $|\gamma|$ is not included entirely both in $B\left(x_{0}, r_{2}\right)$ and $G \backslash B\left(x_{0}, r_{2}\right)$, therefore there exists $y_{1} \in S\left(x_{0}, r_{2}\right) \cap|\gamma|($ see $[\mathrm{Ku}$, Theorem 1, $\S 46$, item I]). Let $\gamma:[0,1] \rightarrow G$ and let $t_{1} \in(0,1)$ be such that $\gamma\left(t_{1}\right)=y_{1}$. There is no loss of generality in assuming that $|\gamma|_{\left[0, t_{1}\right)} \mid \subset B\left(x_{0}, r_{2}\right)$. We put $\gamma_{1}:=\left.\gamma\right|_{\left[0, t_{1}\right)}$. Observe that $\left|\gamma_{1}\right| \subset B\left(x_{0}, r_{2}\right)$, moreover, 
$\gamma_{1}$ is not included entirely either in $\overline{B\left(x_{0}, r_{1}\right)}$ or in $G \backslash \overline{B\left(x_{0}, r_{1}\right)}$. Consequently, there exists $t_{2} \in\left(0, t_{1}\right)$ with $\gamma_{1}\left(t_{2}\right) \in S\left(x_{0}, r_{1}\right)$ (see $[\mathrm{Ku}$, Theorem $1, \S 46$, item I]). There is no loss of generality in assuming that $\left|\gamma_{1}\right|_{\left[t_{2}, t_{1}\right]} \mid \subset G \backslash \overline{B\left(x_{0}, r_{1}\right)}$. Put $\gamma_{2}=$ $\left.\gamma_{1}\right|_{\left[t_{2}, t_{1}\right]}$. Observe that $\gamma_{2}$ is a subcurve of $\gamma$. Hence, $\Gamma\left(C_{1}, C_{0}, G\right)>\Gamma\left(S_{1}, S_{2}, A\right)$ and, consequently, $f\left(\Gamma\left(C_{1}, C_{0}, G\right)\right)>f\left(\Gamma\left(S_{1}, S_{2}, A\right)\right)$. Now, by (1.2),

$$
M_{\alpha^{\prime}}\left(f\left(\Gamma\left(C_{1}, C_{0}, G\right)\right)\right) \leqslant M_{\alpha^{\prime}}\left(f\left(\Gamma\left(S_{1}, S_{2}, A\right)\right)\right) .
$$

Combining (1.3) with (1.7), we obtain (1.5).

We say that the boundary of the domain $G$ is strongly accessible at a point $x_{0} \in \partial G$, if, for every neighborhood $U$ of the point $x_{0}$, there is a compact set $E \subset G$, a neighborhood $V \subset U$ of the point $x_{0}$ and a number $\delta>0$ such that

$$
M_{\alpha}(\Gamma(E, F, G)) \geqslant \delta
$$

for every continuum $F$ in $G$ intersecting $\partial U$ and $\partial V$. We say that the boundary $\partial G$ is strongly accessible, if the corresponding property holds at every point of the boundary.

For instance, so-called $Q E D$-domains in $\mathbf{R}^{n}$ have strongly accessible boundaries, see [MRSY, Remark 13.10]. In particular, the unit ball and half-plane are domains with strongly accessible boundaries, that directly follows from $\left[\mathrm{Vu}_{2}\right.$, Lemma 4.3] and [MRSY, (7.29)]. Consider also the following examples.

Example 1. Let us give an example of a domain with a strongly accessible boundary. Consider the unit disk with a cut. Note that it is a plane domain, whose boundary consists only of finite number of components, and which is finitely connected on the boundary. Applying [ $\mathrm{Na}_{3}$, Theorem 6.2 and Corollary 6.8], we obtain the desired conclusion, see Figure 2 for illustration.

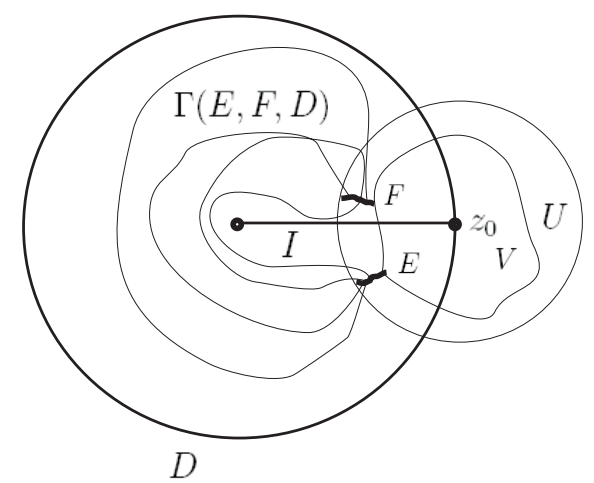

Figure 2. An example of a domain with a strongly accessible boundary.

Example 2. Let us to give an example of domain without strongly accessible boundary. Let $X=\mathbf{R}^{2}$ be equipped with the Euclidean distance $d(x, y)=|x-y|$ and the Lebesgue measure. Consider the square $\Pi=(0,1) \times(0,1)=\{z=(x, y) \in$ $\left.\mathbf{R}^{2}: x \in(0,1), y \in(0,1)\right\}$, on the base of which we construct a desired domain $D$ as follows. Let $I_{k}$ be a segment $I_{k}=\{z=(x, y): x=1 / k, 0 \leqslant y \leqslant 1 / 2\}$, where $k=2,3, \ldots$ is fixed. Put $D:=\prod \backslash \bigcup_{k=2}^{\infty} I_{k}$, see Figure 3 .

We show that $D$ has no strongly accessible boundary, say, at a point $z_{0}=(0,1 / 2)$. By definition, we need to show that there exists a neighborhood $U$ of $z_{0}$ such that, for every neighborhood $V \subset U$, compact $E \subset D$ and a number $\delta>0$ there exists a continuum $F$, intersecting $\partial U$ and $\partial V$, such that

$$
M(\Gamma(E, F, D))<\delta
$$


where $M$ denotes modulus of family of paths in $\mathbf{R}^{2}$. Put $U:=B\left(z_{0}, 1 / 3\right)$, where $z_{0}=(0,1 / 2)$. Fix $V \subset U$, a compact $E \subset D$ and a number $\delta>0$. Let $R_{k}=$ $1 / k, k=1,2, \ldots$. Since $E$ is a compact in $D$, there exists $k_{0}>0$ with $E \cap$ $B\left(z_{0}, R_{k_{0}-1}\right)=\varnothing$. We can consider that all points $z=(x, y) \in E$ satisfy the condition $x>1 /\left(k_{0}-1\right)$. Let $E_{k}$ be a sequence of continua, defined by $E_{k}=$ $\left\{z=(x, y): x=\frac{1}{2}\left(\frac{1}{k}+\frac{1}{(k-1)}\right), \frac{1}{8} \leqslant y \leqslant v_{0}\right\}$, where $v_{0}$ is some positive number with $v_{0}<1 / 2,1 / 2-v_{0}<\operatorname{dist}\left(z_{0}, \partial V\right)$. Let us to show that $E_{k} \cap \partial U \neq \varnothing$ and $E_{k} \cap \partial V \neq \varnothing$ for some $k_{1} \in \mathbf{N}, k_{1}>k_{0}$, and every $k \in \mathbf{N}$. Indeed, let $x_{k}=\left(\frac{1}{2}\left(\frac{1}{k}+\frac{1}{(k-1)}\right), \frac{1}{8}\right)$, $y_{k}=\left(\frac{1}{2}\left(\frac{1}{k}+\frac{1}{(k-1)}\right), v_{0}\right)$ are the "lowest" and the "highest" points of $E_{k}$, correspondingly. Now $\left|z_{0}-x_{k}\right| \rightarrow 3 / 8, \quad\left|z_{0}-y_{k}\right| \rightarrow 1 / 2-v_{0}, \quad k \rightarrow \infty$. Thus, $x_{k} \notin B\left(z_{0}, 1 / 3\right)=U$ and $y_{k} \in V$ for sufficiently large $k>k_{0}$. Now, $E_{k} \cap(D \backslash U) \neq \varnothing$ and $E_{k} \cap V \neq \varnothing$ for $k>k_{0}$. Now, by [Ku, Theorem 1.I.5, $\left.\S 46\right]$ we obtain that $E_{k} \cap \partial U \neq \varnothing$ and $E_{k} \cap \partial V \neq \varnothing$. Let $k_{1}$ be a smallest $k \geqslant k_{0}$, for which above relations hold.

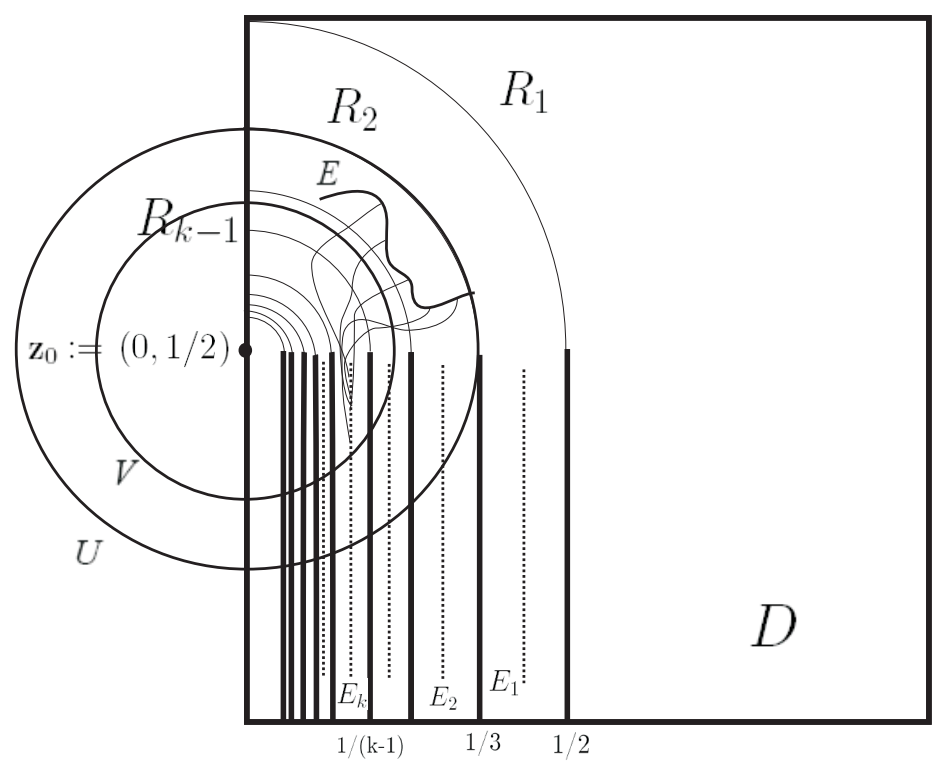

Figure 3. An example of a domain without a strongly accessible boundary.

Let $L_{k}$ be a sector of the disk $B\left(z_{0}, R_{k-1}\right)$, defined by $L_{k}=\{z=(x, y)=$ $\left.z_{0}+r e^{i \varphi}: 0<r<R_{k-1}, \varphi \in[0, \pi / 2)\right\}$. Put $P_{k}=\{z=(x, y) \in D: 0<x<$ $1 /(k-1), 0<y \leqslant 1 / 2\}$ and $D_{k}:=L_{k} \cup P_{k}$. It is clear, that $D_{k}$ is a domain, and that $E_{k} \subset D_{k}$. By construction, $E \subset D \backslash D_{k}$ for $k>k_{0}$.

Let $\Gamma_{k}$ be a family of paths, joining $E_{k}$ and $E$ in $D$. In what follows, $|\gamma|$ is a locus of $\gamma$. If $\gamma \in \Gamma_{k}$, then $|\gamma| \cap D_{k} \neq \varnothing \neq|\gamma| \cap\left(D \backslash D_{k}\right)$. Now, by [Ku, Theorem 1.I.5, $\S 46]$ we obtain that $|\gamma| \cap \partial D_{k} \neq \varnothing$. Since the path $\gamma$ belongs to $D$, we conclude that there exists subpath $\gamma_{1}<\gamma$, joining $S\left(z_{0}, R_{k-1}\right)$ and $E, k>k_{0}$. Similarly, we may show that $\gamma$ has a subpath, joining $S\left(z_{0}, R_{k-1}\right)$ and $S\left(z_{0}, R_{k_{0}-1}\right)$ in $\mathbf{R}^{2}$. Thus,

$$
\Gamma_{k}>\Gamma\left(S\left(z_{0}, R_{k-1}\right), S\left(z_{0}, R_{k_{0}-1}\right), \mathbf{R}^{2}\right), \quad k>k_{0} .
$$

By [Va, Theorem 7.5]

$$
M\left(\Gamma\left(S\left(x_{0}, R_{k-1}\right), S\left(x_{0}, R_{k_{0}-1}\right), \mathbf{R}^{2}\right)\right)=\frac{2 \pi}{\log \frac{R_{k_{0}-1}}{R_{k-1}}} \rightarrow 0, \quad k \rightarrow \infty .
$$


By (1.9) and (1.10) we obtain that $M\left(\Gamma\left(E, E_{k}, D\right)\right) \rightarrow 0, k \rightarrow \infty$. From here, we obtain that there exists $k_{2}>k_{1}$ such that $M\left(\Gamma\left(E, E_{k}, D\right)\right)<\delta$. Put $F=E_{k_{2}}$. By properties of $E_{k}, k>k_{1}$, established above, $F \cap \partial U \neq \varnothing$ and $F \cap \partial V \neq \varnothing$. Moreover, $F$ satisfies (1.8). Thus, boundary of $D$ is not strongly accessible at $z_{0}$.

Let $X$ and $Y$ be metric spaces. A mapping $f: X \rightarrow Y$ is discrete if $f^{-1}(y)$ is discrete for all $y \in Y$ and $f$ is open if it takes open sets onto open sets. Given a domain $D \subset X$, the cluster set of $f: D \rightarrow Y$ at $b \in \partial D$ is the set $C(f, b)$ of all points $z \in Y$ for which there exists a sequence $\left\{b_{k}\right\}_{k=1}^{\infty}$ in $D$ such that $b_{k} \rightarrow b$ and $f\left(b_{k}\right) \rightarrow z$ as $k \rightarrow \infty$. For a non-empty set $E \subset \partial D$ let $C(f, E)=\bigcup C(f, b)$, where $b$ ranges over set $E$. A mapping $f: G \rightarrow Y$ is closed in $G \subset X$ if $f(A)$ is closed in $f(G)$ whenever $A$ closed in $G$. A mapping $f$ is proper if $f^{-1}(K)$ is compact in $D$ whenever $K$ is a compact set of $f(D)$. A mapping $f$ is boundary preserving if $C(f, \partial D) \subset \partial f(D)$.

Let $D \subset X, f: D \rightarrow X^{\prime}$ be a discrete open mapping, $\beta:[a, b) \rightarrow X^{\prime}$ be a curve, and $x \in f^{-1}(\beta(a))$. A curve $\alpha:[a, c) \rightarrow D$ is called a maximal $f$-lifting of $\beta$ starting at $x$, if $(1) \alpha(a)=x$; (2) $f \circ \alpha=\left.\beta\right|_{[a, c)}$; (3) for $c<c^{\prime} \leqslant b$, there are no curves $\alpha^{\prime}:\left[a, c^{\prime}\right) \rightarrow D$ such that $\alpha=\left.\alpha^{\prime}\right|_{[a, c)}$ and $f \circ \alpha^{\prime}=\left.\beta\right|_{\left[a, c^{\prime}\right)}$. In the case $X=X^{\prime}=\mathbf{R}^{n}$, the assumption on $f$ yields that every curve $\beta$ with $x \in f^{-1}(\beta(a))$ has a maximal $f$-lifting starting at $x$ (see [Ri, Corollary II.3.3], [MRV, Lemma 3.12]). Consider the condition

A: for all $\beta:[a, b) \rightarrow X^{\prime}$ and $x \in f^{-1}(\beta(a))$, a mapping $f: D \rightarrow X^{\prime}$ has a maximal $f$-lifting in $D$ starting at $x$.

Remark 1.2. As noted above, the condition $\mathbf{A}$ holds in $\mathbf{R}^{n}$ for open discrete mappings. We give some examples of other spaces with a similar property.

1) Riemannian manifolds. The existence of maximal liftings under open discrete mappings of a domain of a Riemannian manifold into another Riemannian manifold is established in [IS, Proposition 2.1].

2) Riemannian surfaces. The existence of maximal liftings for open discrete mappings may be obtained similarly to item $\mathbf{1}$ ).

3) Carnot and Heisenberg groups. We refer the reader to the results of works [MV, Theorem 3.6] and [UV, Lemma 5]. Although these results apply only to particular cases of open discrete mappings, the general case can be proved similarly.

In a more general situation, the existence of maximal liftings under open discrete mappings of arbitrary orientable topological manifolds is established in [OR, Theorem 3.4], cf. [HR, Example 1.4(a)].

Let $G$ be a domain in a space $(X, d, \mu)$. Similarly to [IR], we say that a function $\varphi: G \rightarrow \mathbf{R}$ has finite mean oscillation at a point $x_{0} \in \bar{G}$, abbr. $\varphi \in F M O\left(x_{0}\right)$, if

$$
\varlimsup_{\varepsilon \rightarrow 0} \frac{1}{\mu\left(B\left(x_{0}, \varepsilon\right)\right)} \int_{B\left(x_{0}, \varepsilon\right)}\left|\varphi(x)-\bar{\varphi}_{\varepsilon}\right| d \mu(x)<\infty
$$

where

$$
\bar{\varphi}_{\varepsilon}=\frac{1}{\mu\left(B\left(x_{0}, \varepsilon\right)\right)} \int_{B\left(x_{0}, \varepsilon\right)} \varphi(x) d \mu(x)
$$

is the mean value of the function $\varphi(x)$ over the set $B\left(x_{0}, \varepsilon\right)=\left\{x \in G: d\left(x, x_{0}\right)<\varepsilon\right\}$ with respect to the measure $\mu$. Here the condition (1.11) includes the assumption that $\varphi$ is integrable with respect to the measure $\mu$ over the set $B\left(x_{0}, \varepsilon\right)$ for some $\varepsilon>0$. 
Remark 1.3. Some examples of functions of finite mean oscillation in Euclidean space are well known. For example, the function $\varphi(x)=\log \frac{1}{\left|x-x_{0}\right|}$ has a finite mean oscillation at every point $x_{0} \in \mathbf{R}^{n}$, see e.g. [RR, p. 5]. On this basis, it is not difficult to construct at least three simple examples of such functions in more general metric spaces.

1) Riemannian manifolds. Let $d(x, y)$ be a geodesic distance in a Riemannian manifold $\mathbf{M}^{n}, n \geqslant 2$, see [IS]. Now, we put $\varphi_{1}(x):=\log \frac{1}{d\left(x, x_{0}\right)}$. Since in so-called normal coordinates $(\psi, U)$ of a neighborhood $U$ of the point $x_{0}$ the geodesic distance $d\left(x, x_{0}\right)$ equals to $\left|\psi(x)-\psi\left(x_{0}\right)\right|, x \in U$, the fact that the function $\varphi_{1}$ belongs to $F M O\left(x_{0}\right)$ follows from the corresponding result for Euclidean space.

2) Hyperbolic space. Let $\mathbf{D}$ be the unit disk on the plane. The hyperbolic distance in the unit disk $\mathbf{D}$ is given by the formula

$$
h\left(z_{1}, z_{2}\right)=\log \frac{1+t}{1-t}, \quad t=\frac{\left|z_{1}-z_{2}\right|}{\left|1-z_{1} \overline{z_{2}}\right|},
$$

while the hyperbolic area of a set $S$ in $\mathbf{D}$ is calculated as the integral $h(S)=$ $\int_{S} \frac{4 d m(z)}{\left(1-|z|^{2}\right)^{2}}$, see e.g. [RV, (2.4)-(2.5)]. Given a Borel function $\rho: \mathbf{D} \rightarrow[0, \infty]$, a Lebesgue measurable set $S \subset \mathbf{D}$ and a locally rectifiable path $\gamma:(a, b) \rightarrow \mathbf{D}$ we set

$$
\int_{S} \rho(z) d h(z):=\int_{S} \frac{4 \rho(z) d m(z)}{\left(1-|z|^{2}\right)^{2}}, \quad \int_{\gamma} \rho(z) d s_{h}(z):=\int_{\gamma} \frac{2 \rho(z)|d z|}{1-|z|^{2}} .
$$

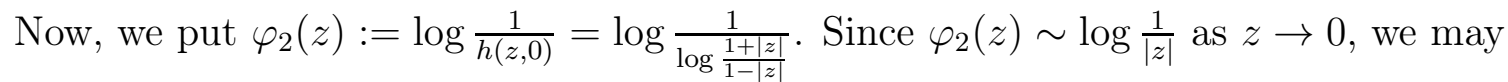
obtain a two-sided estimate $C_{1} \cdot \log \frac{1}{|z|} \leqslant \varphi_{2}(z) \leqslant C_{2} \cdot \log \frac{1}{|z|}$ in some neighborhood $U$ of the origin under some constants $C_{1}, C_{2}>0$. Putting $B_{h}(0, \varepsilon):=\{x \in \mathbf{D}: h(x, 0)<$ $\varepsilon\}$, we observe that $h\left(B_{h}(0, r)\right)=\pi\left(e^{r / 2}-e^{-r / 2}\right)^{2}$, see [Berd, Theorem 7.2.2]. Since $e^{r / 2}-e^{-r / 2} \sim r$ as $r \rightarrow 0$, there exist constants $C_{3}, C_{4}>0$ such that $C_{3} \pi r^{2} \leqslant$ $h\left(B_{h}(0, r)\right) \leqslant C_{4} \pi r^{2}$ for sufficiently small $r>0$. Finally, the function $4 /\left(1-|z|^{2}\right)^{2}$ is bounded from below and from above as $z \rightarrow 0$, thus, $C_{5} \leqslant 4 /\left(1-|z|^{2}\right)^{2} \leqslant C_{6}$ in some neighborhood of the origin for some positive constants $C_{5}$ and $C_{6}$. Using the definition of $F M O$ in (1.11), let us to show that $\varphi_{2}(z) \in F M O(0)$. Given $\varepsilon>0$ sufficiently small, we obtain that

$$
\begin{aligned}
\frac{C_{5} \cdot C_{1}}{\pi \varepsilon^{2} \cdot C_{4}} \int_{B_{h}(0, \varepsilon)} \log \frac{1}{|z|} d m(z) & \leqslant \bar{\varphi}_{2}=\frac{1}{h\left(B_{h}(0, \varepsilon)\right)} \int_{B_{h}(0, \varepsilon)} \varphi_{2}(z) d h(z) \\
& \leqslant \frac{C_{6} \cdot C_{2}}{\pi \varepsilon^{2} \cdot C_{3}} \int_{B_{h}(0, \varepsilon)} \log \frac{1}{|z|} d m(z),
\end{aligned}
$$

where $d h(z)=\frac{4 d m(z)}{\left(1-|z|^{2}\right)^{2}}$. Setting $\bar{\varphi}_{\varepsilon}:=\frac{1}{\pi \varepsilon^{2}} \int_{B_{h}(0, \varepsilon)} \log \frac{1}{|z|} d m(z)$, we obtain that $\varphi_{2}(z)-$ $\overline{\varphi_{2}} \varepsilon \leqslant \widetilde{C_{1}}\left|\log \frac{1}{|z|}-\bar{\varphi}_{\varepsilon}\right|$, where $\widetilde{C_{1}}=\max \left\{C_{2},\left(C_{5} \cdot C_{1}\right) / C_{4}\right\}$. Similarly, ${\overline{\varphi_{2}}}_{\varepsilon}-\varphi_{2}(z) \leqslant$ $\widetilde{C_{2}}\left|\log \frac{1}{|z|}-\bar{\varphi}_{\varepsilon}\right|$, where $\widetilde{C_{2}}=\max \left\{C_{1},\left(C_{6} \cdot C_{2}\right) / C_{3}\right\}$. Let $C=\max \left\{\widetilde{C_{1}}, \widetilde{C_{2}}\right\}$. Now

$$
\frac{1}{h\left(B_{h}(0, \varepsilon)\right)} \int_{B_{h}(0, \varepsilon)}\left|\varphi_{2}(z)-\bar{\varphi}_{2}\right| d h(z) \leqslant \frac{C \cdot C_{6}}{C_{3} \cdot \pi \varepsilon^{2}} \int_{B\left(0, \frac{e^{\varepsilon}-1}{e^{\varepsilon}+1}\right)}\left|\log \frac{1}{|z|}-\bar{\varphi}_{\varepsilon}\right| d m(z) .
$$

Here we have used that $B_{h}(0, \varepsilon)=B\left(0, \frac{e^{\varepsilon}-1}{e^{\varepsilon}+1}\right)$ and $\frac{e^{\varepsilon}-1}{e^{\varepsilon}+1} \sim \varepsilon$ as $\varepsilon \rightarrow 0$. By [RR, p. 5], $\varphi_{2}(z) \in F M O(0)$. 
3) Riemannian surfaces. The Poincaré uniformization theorem (1908) states that every Riemann surface $\mathbf{S}$ is represented (up to the conformal equivalence) in the form of the factor $\widetilde{\mathbf{S}} / G$, where $\widetilde{\mathbf{S}}$ is one of the canonical domains: $\mathbf{C}, \overline{\mathbf{C}}$ or the unit disk $\mathbf{D}$ in $\mathbf{C}$ and $G$ is a discrete group of conformal (= fractional) mappings of $\mathbf{S}$ onto itself. Assume that $\mathbf{S}$ has a hyperbolic type, that is, $\widetilde{\mathbf{S}}=\mathbf{D}$. Moreover, assume that $\mathbf{S}=\mathbf{D} / G$. Note that $\mathbf{D} / G$ is a Riemannian surface, see e.g. [Berd, Theorem 6.2.1].

Let $p_{1}$ and $p_{2} \in \mathbf{D} / G$. Then by the definition $p_{1}$ and $p_{2}$ are orbits $G_{z_{1}}$ and $G_{z_{2}}$ of points $z_{1}$ and $z_{2} \in \mathbf{D}$, see $[R V]$. Set

$$
\widetilde{h}\left(p_{1}, p_{2}\right):=\inf _{g_{1}, g_{2} \in G} h\left(g_{1}\left(z_{1}\right), g_{2}\left(z_{2}\right)\right),
$$

where $h$ is a hyperbolic metric in $\mathbf{D}$. Observe that $\widetilde{h}$ is a metric on $\mathbf{D} / G$ (see [RV]).

Let $\pi: \mathbf{D} \rightarrow \mathbf{D} / G$ be the natural projection. Putting $p_{0} \in \mathbf{D} / G$, we can find $z_{0} \in$ D such that $\pi\left(z_{0}\right)=p_{0}$. Let $D_{0}$ be Dirichlet polygon for $G$ centered at $z_{0}$. Without loss of generality, we can consider that $z_{0}=0$. Observe that $\left.\pi\right|_{D_{0}}$ is a homeomorphism of $D_{0}$ onto some open set $U_{0} \subset \mathbf{D} / G$, cf. [Berd, Proposition 9.2.2(iv)]. Putting $\psi=\left(\left.\pi\right|_{D_{0}}\right)^{-1}$, we define the area $\widetilde{h}$ of a set $E \subset U_{0}$ by $\widetilde{h}(E)=\int_{\psi(E)} \frac{4 d m(z)}{\left(1-|z|^{2}\right)^{2}}$. We can find a neighborhood $V_{0} \subset U_{0}$ of $p_{0}$ such that $\widetilde{h}\left(p, p_{0}\right)=h(\psi(p), 0)$ for every $p \in V_{0}$, where $h$ is a hyperbolic metric in $\mathbf{D}$. Now, we put $\varphi_{3}(p):=\log \frac{1}{h(\psi(p), 0)}$. Using item 2), we obtain that $\varphi_{3} \in F M O\left(p_{0}\right)$.

The following result holds.

Theorem 1.1. Let $D$ and $D^{\prime}$ be domains with finite Hausdorff dimensions $\alpha$ and $\alpha^{\prime} \geqslant 2$ in spaces $(X, d, \mu)$ and $\left(X^{\prime}, d^{\prime}, \mu^{\prime}\right)$, respectively. Assume that $X$ is complete and supports an $\alpha$-Poincaré inequality, and that the measure is doubling. Let $D$ be a bounded domain which is finitely connected at the boundary, and let $Q: X \rightarrow(0, \infty)$ be a locally integrable function. Suppose that $f: D \rightarrow D^{\prime}, D^{\prime}=$ $f(D)$, is a discrete, closed and open ring $Q$-mapping in $\partial D$, for which A-condition holds. Moreover, suppose that $\partial D^{\prime}$ is strongly accessible and $\overline{D^{\prime}}$ is compact in $X^{\prime}$. Then $f$ has a continuous extension $f: \bar{D}_{P} \rightarrow \overline{D^{\prime}}, f\left(\bar{D}_{P}\right)=\overline{D^{\prime}}$, whenever $Q \in F M O(\partial D)$.

By correspondence $\left[E_{k}\right] \mapsto f\left(\left[E_{k}\right]\right),\left[E_{k}\right] \in E_{D}, f\left(\left[E_{k}\right]\right) \in \partial D^{\prime}$, we mean the following. If $\left\{x_{k}\right\}_{k=1}^{\infty}$ is a sequence with $x_{k} \rightarrow\left[E_{k}\right], k \rightarrow \infty$, then we set: $f\left(\left[E_{k}\right]\right):=$ $\lim _{k \rightarrow \infty} f\left(x_{k}\right)$. The statement of the Theorem 1.1 includes that this limit exists, and it does not depend on a sequence $\left\{x_{k}\right\}_{k=1}^{\infty}$, which converges to $\left[E_{k}\right]$ (see the Figure 4 ).
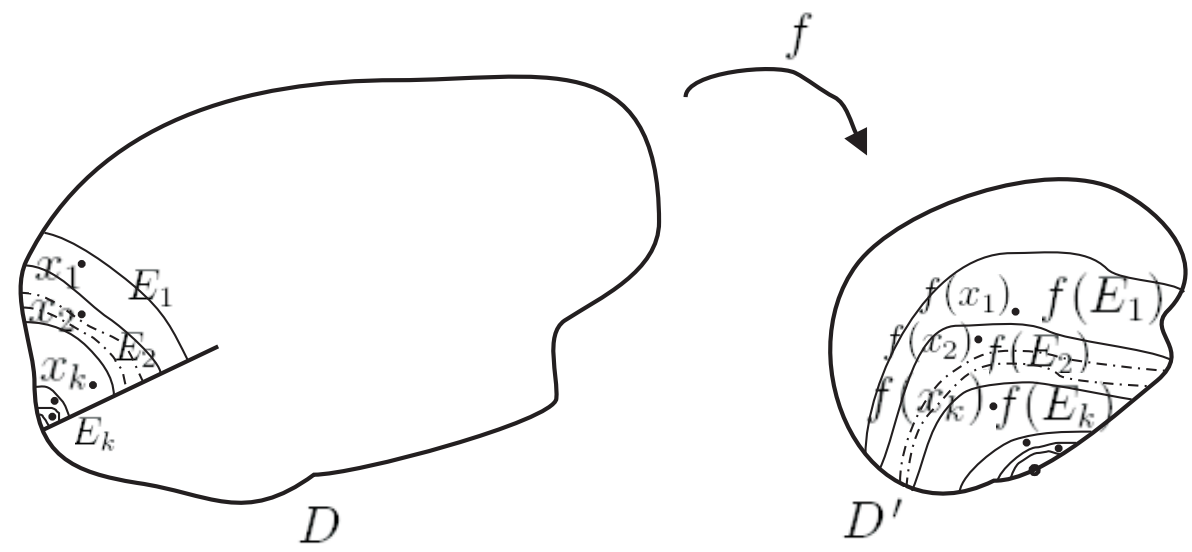

Figure 4. A correspondence of prime ends and boundary points under a mapping. 
Remark 1.4. Theorem 1.1, presented above, generalizes [A, Theorem 5], that corresponds to the case of bounded function $Q$ in (1.3). Together with Example 5 in [A], this theorem shows that homeomorphisms with the condition $M_{\alpha}(\Gamma) / K \leqslant$ $M_{\alpha}(f(\Gamma)) \leqslant K \cdot M_{\alpha}(\Gamma)$ have continuous boundary extension in terms of prime ends. Note that, the estimate of the modulus of families of paths used here, unlike (1.3), is two-sided. On this point, cf. [A, Theorems 4, 5 and Corollary 2] and Theorems 1.1 and 4.2 .

Remark 1.5. In fact, the FMO type condition that is present in Theorem 1.1 can be replaced by the following more general and more fundamental assumption, which will be used later in proving all the main results. Given a point $x_{0} \in \bar{D}$, assume that there exists a Lebesgue measurable function $\psi:(0, \infty) \rightarrow(0, \infty)$ such that

$$
I\left(\varepsilon, \varepsilon_{0}\right):=\int_{\varepsilon}^{\varepsilon_{0}} \psi(t) d t<\infty
$$

for every $\varepsilon \in\left(0, \varepsilon_{0}\right)$ and $I\left(\varepsilon, \varepsilon_{0}\right) \rightarrow \infty$ as $\varepsilon \rightarrow 0$, and

$$
\int_{\varepsilon<d\left(x, x_{0}\right)<\varepsilon_{0}} Q(x) \cdot \psi^{\alpha}\left(d\left(x, x_{0}\right)\right) d \mu(x)=o\left(I^{\alpha}\left(\varepsilon, \varepsilon_{0}\right)\right), \quad \varepsilon \rightarrow 0 .
$$

\section{Main Lemma}

The following statement holds (see also $\left[\mathrm{Vu}_{1}\right.$, Theorem 3.3] for space $\mathbf{R}^{n}$ ).

Proposition 2.1. Let $(X, d, \mu)$ be metric space with Borel measure $\mu$, and let $D$ be a domain in $X$. Assume that the measure is doubling. If $f: D \rightarrow X^{\prime}$ is a discrete, closed and open mapping of $D$ onto a set $D^{\prime}$, then $f$ is boundary preserving. Moreover, $f^{-1}(K)$ is a compact for every compact set $K \subset D^{\prime}$.

Proof. Since $f$ is open, $D^{\prime}$ is a domain. Assume, to the contrary, that $f$ is not boundary preserving. Then there exists $x_{0} \in \partial D$ and $y \in D^{\prime}$ such that $y \in C\left(f, x_{0}\right)$. Now, we can find a sequence $x_{k} \rightarrow x_{0}$ as $k \rightarrow \infty, x_{k} \in D, k=1,2, \ldots$, such that $f\left(x_{k}\right) \rightarrow y$ as $k \rightarrow \infty$.

Without loss of generality, we can consider that $f\left(x_{k}\right) \neq y$ for all $k=1,2 \ldots$ In fact, by continuity of $f$, for every $k \in \mathbf{N}$ there exists $\delta_{k}>0$ such that

$$
d^{\prime}\left(f(x), f\left(x_{k}\right)\right)<1 / k \quad \forall x \in B\left(x_{k}, \delta_{k}\right) .
$$

We can consider that $B\left(x_{k}, \delta_{k}\right) \subset D$ and $\delta_{k}<1 / k$. Let $r \rightarrow 0$ in (1.1) at $y=$ $x=x_{0}$. Now, we obtain that $\mu\left(\left\{x_{k}\right\}\right)=0$. Fix $i \in \mathbf{N}$. Since, by assumption on $\mu, \mu\left(B\left(x_{k}, \delta_{k} / 2^{i}\right)\right)>0$, we obtain that $B\left(x_{k}, \delta_{k} / 2^{i}\right)$ contains at least two points. By increasing of $i, i=1,2, \ldots$, we obtain a sequence $x_{i k} \in B\left(x_{k}, \delta_{k} / 2^{i}\right)$ such that $x_{i k} \rightarrow x_{k}$ as $i \rightarrow \infty$ and $x_{i k} \neq x_{k}$ for every $i \in \mathbf{N}$. By discreteness of $f$, we can consider that $f\left(x_{i k}\right) \neq y_{0}$ for all $i \in \mathbf{N}$. Fix some such $i_{0} \in \mathbf{N}$ and set $z_{k}:=x_{i_{0} k}$. Now, by the triangle inequality,

$$
d\left(z_{k}, x_{0}\right) \leqslant d\left(z_{k}, x_{k}\right)+d\left(x_{k}, x_{0}\right) \rightarrow 0, \quad k \rightarrow \infty
$$

and, simultaneously, by (2.1)

$$
d^{\prime}\left(f\left(z_{k}\right), y\right) \leqslant d^{\prime}\left(f\left(z_{k}\right), f\left(x_{k}\right)\right)+d^{\prime}\left(f\left(x_{k}\right), y\right)<1 / k+d^{\prime}\left(f\left(x_{k}\right), y\right) \rightarrow 0, \quad k \rightarrow \infty .
$$

So, $z_{k} \in D, z_{k} \rightarrow x_{0}$ as $k \rightarrow \infty, f\left(z_{k}\right) \rightarrow y$ as $k \rightarrow \infty$, and $f\left(z_{k}\right) \neq y$ for every $k \in \mathbf{N}$. On the other hand, note that $\left\{x_{k}\right\}_{k=1}^{\infty}$ is closed in $D$, but $\left\{f\left(x_{k}\right)\right\}_{k=1}^{\infty}$ is not closed in $f(D)$, because $y \notin\left\{f\left(x_{k}\right)\right\}_{k=1}^{\infty}$. Now $f$ is not closed in $D$. This contradicts assumptions of the proposition and disproves that $f$ is not boundary preserving. 
It remains to show that $f^{-1}(K)$ is a compact for every compact set $K \subset D^{\prime}$. If this is not true, there exists a sequence $x_{k} \in f^{-1}(K)$, such that $x_{k} \rightarrow x_{0} \in \partial D$. As was shown above, $f\left(x_{k}\right) \rightarrow y_{0} \in \partial D^{\prime}$, which contradicts condition $x_{k} \in f^{-1}(K)$.

The following statement was proved in [GRY, Lemma 5.1] for homeomorphisms in $\mathbf{R}^{2}$.

Lemma 2.1. Suppose that the assumptions of Theorem 1.1 are satisfied. Moreover, assume that, for every $x_{0} \in \partial D$ conditions (1.15)-(1.16) hold. Then $f$ has a continuous extension $f: \bar{D}_{P} \rightarrow \overline{D^{\prime}}, f\left(\bar{D}_{P}\right)=\overline{D^{\prime}}$.

Proof. By Proposition 1.2, $\bar{D}_{P}$ is metrizable. Now, by metrizability of $\bar{D}_{P}$, it is sufficient to prove that

$$
L=C(f, P):=\left\{y \in X^{\prime}: y=\lim _{k \rightarrow \infty} f\left(x_{k}\right), x_{k} \rightarrow P, x_{k} \in D\right\}
$$

consists of single point $y_{0} \in \partial D^{\prime}$. Since $\overline{D^{\prime}}$ is a compact, $L \neq \varnothing$. By Proposition 2.1, $L \subset \partial D^{\prime}$.

Assume, to the contrary, that $f$ cannot be extended to $P$ continuously. Now, we can find at least two points $y_{0}$ and $z_{0} \in L$. Set $U=B\left(y_{0}, r_{0}\right)$, where $0<r_{0}<$ $d\left(y_{0}, z_{0}\right)$. Now we can find a sequences $y_{k}$ and $z_{k}$ in $f\left(E_{k}\right), k=1,2, \ldots, P=\left[E_{k}\right]$, such that $d\left(y_{0}, y_{k}\right)<r_{0}$ and $d\left(y_{0}, z_{k}\right)>r_{0}$ and, besides that, $y_{k} \rightarrow y_{0}$ and $z_{k} \rightarrow z_{0}$ as $k \rightarrow \infty$. By Remark 4.5 in [ABBS] we can consider that the sets $E_{k}$ are open. Moreover, by Remark 2.6 in [ABBS] the set $E_{k}$ is path connected for every $k \in \mathbf{N}$.

Denote $x_{0}:=I\left(\left[E_{k}\right]\right)$ (see Proposition 1.1). Now we show that, for every $r>0$ there exists $k \in \mathbf{N}$ such that

$$
E_{k} \subset B\left(x_{0}, r\right) \cap D .
$$

Assume, to the contrary, that there exists $r>0$ with the following condition: for every $k \in \mathbf{N}$ there exists $x_{k} \in E_{k} \backslash B\left(x_{0}, r\right)$. Since $\mu$ is doubling, $X$ is complete if and only if it is proper (i.e. every closed bounded set is compact), see [BB, Proposition 3.1]. Since $D$ is bounded, $\bar{D}$ is compact. Now, we can find a subsequence $x_{k_{l}} \in D$ with $x_{k_{l}} \rightarrow \overline{x_{0}}$ as $l \rightarrow \infty$ for some $\overline{x_{0}} \in \bar{D}$. Given $i \in \mathbf{N}$, there exists $l_{0} \in \mathbf{N}$ such that $k_{l}>i$ for every $l \geqslant l_{0}$. Consequently, $x_{k_{l}} \in E_{k_{l}} \subset E_{i}$ for every $l \geqslant l_{0}$ and thus, $\overline{x_{0}} \in \overline{E_{i}}$. Since $i$ is arbitrary, we obtain that $\overline{x_{0}} \in \bigcap_{i=1}^{\infty} \overline{E_{i}}=\left\{x_{0}\right\}$. So, $x_{0}=\overline{x_{0}}$. It remains to show that $x_{k} \rightarrow x_{0}$ as $k \rightarrow \infty$. Assume the contrary, then there exists a subsequence $x_{m_{l}} \in D$ with $x_{m_{l}} \rightarrow \zeta_{0}$ as $l \rightarrow \infty$. Arguing as above, we obtain that $\zeta_{0}=x_{0}$, that disproves the contradiction mentioned above. Now $x_{k} \rightarrow x_{0}$ as $k \rightarrow \infty$ and thus, $x_{k} \in B\left(x_{0}, r\right)$. The inclusion (2.2) have been proved.

Since $y_{k}, z_{k} \in f\left(E_{k}\right)$, one can find at least two sequences $x_{k}, x_{k}^{\prime} \in E_{k}$ such that $f\left(x_{k}\right)=y_{k}$ and $f\left(x_{k}^{\prime}\right)=z_{k}$. By $(2.2) x_{k} \rightarrow x_{0}$ and $x_{k}^{\prime} \rightarrow x_{0}$ as $k \rightarrow \infty$. According to the definition of a strongly accessible boundary at a point $y_{0} \in \partial D^{\prime}$, for any neighborhood $U$ of this point one can find a compact set $C_{0} \subset \partial D^{\prime}$, a neighborhood $V$ of the point $y_{0}$ and a number $\delta>0$ such that

$$
M_{\alpha^{\prime}}\left(\Gamma\left(C_{0}^{\prime}, F, D^{\prime}\right)\right) \geqslant \delta>0
$$

for an arbitrary continuum $F$ that intersects $\partial U$ and $\partial V$. By Proposition 2.1, $C:=$ $f^{-1}\left(C_{0}^{\prime}\right)$ is compact subset of $D$. Consequently, $\delta_{0}=\operatorname{dist}\left(x_{0}, C\right)>0$. Then, without loss of generality, we can assume that $C_{0} \cap \overline{B\left(x_{0}, \varepsilon_{0}\right)}=\varnothing$. Since $E_{k}$ is connected, the points $x_{k}$ and $x_{k}^{\prime}$ can be connected by a curve $\gamma_{k}$ lying in $E_{k}$. Since $f\left(x_{k}\right)=y_{k} \in V$ and $f\left(x_{k}^{\prime}\right)=z_{k} \in D^{\prime} \backslash U$ for sufficiently large $k \in \mathbf{N}$, one can find a number $k_{0} \in \mathbf{N}$ 
such that, by virtue of (2.3),

$$
M_{\alpha^{\prime}}\left(\Gamma\left(C_{0}^{\prime}, f\left(\gamma_{k}\right), D^{\prime}\right)\right) \geqslant \delta>0
$$

for all $k \geqslant k_{0}$. Let $\Gamma_{k}$ denote the family of all semiopen curves $\beta_{k}:[a, b) \rightarrow D^{\prime}$ such that $\beta(a) \in f\left(\gamma_{k}\right), \beta_{k}(t) \in D^{\prime}$ for all $t \in[a, b)$, and

$$
\lim _{t \rightarrow b-0} \beta_{k}(t):=B_{k} \in C_{0}^{\prime} \text {. }
$$

It is obvious that

$$
M_{\alpha^{\prime}}\left(\Gamma_{k}\right)=M_{\alpha^{\prime}}\left(\Gamma\left(C_{0}^{\prime}, f\left(\gamma_{k}\right), D^{\prime}\right)\right) .
$$

For each fixed $k \in \mathbf{N}, k \geqslant k_{0}$, we consider the family $\Gamma_{k}^{\prime}$ of maximal liftings $\alpha_{k}(t):[a, c) \rightarrow D$ of the family $\Gamma_{k}$ with origin in the set $\gamma_{k}$. This family exists and is well defined by virtue of A-condition. First, note that no curve $\alpha_{k}(t) \in \Gamma_{k}^{\prime}$, $\alpha_{k}:[a, c) \rightarrow D$, can not tend to the boundary of the domain $D$ as $t \rightarrow c-0$ by virtue of the condition $C(f, \partial D) \subset \partial D^{\prime}$. Then $C\left(\alpha_{k}(t), c\right) \subset D$. Now assume that the curve $\alpha_{k}(t)$ does not have a limit as $t \rightarrow c-0$.

Consider

$$
G=\left\{x \in X: x=\lim _{k \rightarrow \infty} \alpha\left(t_{k}\right)\right\}, \quad t_{k} \in[a, c), \quad \lim _{k \rightarrow \infty} t_{k}=c .
$$

Letting to subsequences, if it is need, we can restrict us by monotone sequences $t_{k}$. For $x \in G$, by continuity of $f, f\left(\alpha\left(t_{k}\right)\right) \rightarrow f(x)$ as $k \rightarrow \infty$, where $t_{k} \in[a, c), t_{k} \rightarrow c$ as $k \rightarrow \infty$. However, $f\left(\alpha\left(t_{k}\right)\right)=\beta\left(t_{k}\right) \rightarrow \beta(c)$ as $k \rightarrow \infty$. Thus, $f$ is a constant on $G$. On the other hand, $\bar{\alpha}$ is a compact set, because $\bar{\alpha}$ is a closed subset of the compact space $\bar{D}$ (see $[\mathrm{Ku}$, Theorem 2.II.4, $\S 41]$ ). Now, by Cantor condition on the compact $\bar{\alpha}$, by monotonicity of $\alpha\left(\left[t_{k}, c\right)\right)$,

$$
G=\bigcap_{k=1}^{\infty} \overline{\alpha\left(\left[t_{k}, c\right)\right)} \neq \varnothing,
$$

see $[\mathrm{Ku}, 1 . I I .4, \S 41]$. Now, by [Ku, Theorem 5.II.5, $\S 47], \bar{\alpha}$ is connected. By discreteness of $f, G$ is a single-point set, and $\alpha:[a, c) \rightarrow D$ extends to a closed curve $\alpha:[a, c] \rightarrow D$, and $f(\alpha(c))=\beta(c)$.

Therefore, there exists $\lim _{t \rightarrow c-0} \alpha_{k}(t)=A_{k} \in D$. Observe that, in this case, by the definition of maximal lifting, we have $c=b$. Then, on the one hand, $\lim _{t \rightarrow b-0} \alpha_{k}(t):=A_{k}$, and, on the other hand, by virtue of the continuity of the mapping $f$ in $D$,

$$
f\left(A_{k}\right)=\lim _{t \rightarrow b-0} f\left(\alpha_{k}(t)\right)=\lim _{t \rightarrow b-0} \beta_{k}(t)=B_{k} \in C_{0}^{\prime} .
$$

According to the definition of $C_{0}$, this implies that $A_{k}$ belongs to $C_{0}$. We imbed the compact set $C_{0}$ into a certain continuum $C_{1}$ lying completely in the domain $D$ (see Lemma 1 in $[\mathrm{Sm}]$ ). Taking a smaller value of $\varepsilon_{0}>0$, we can again assume that $C_{1} \cap \overline{B\left(x_{0}, \varepsilon_{0}\right)}=\varnothing$. Now we have that $\Gamma_{k}^{\prime} \subset \Gamma\left(\gamma_{k}, C_{1}, D\right)$. Passing to a subsequence, if necessary, we can consider that $x_{k}$ and $x_{k}^{\prime} \in B\left(x_{0}, 2^{-k}\right)$. Observe that the function

$$
\eta(t)= \begin{cases}\psi(t) / I\left(2^{-k}, \varepsilon_{0}\right), & t \in\left(2^{-k}, \varepsilon_{0}\right), \\ 0, & t \in \mathbf{R} \backslash\left(2^{-k}, \varepsilon_{0}\right),\end{cases}
$$

where $I(\varepsilon):=\int_{\varepsilon}^{\varepsilon_{0}} \psi(t) d t$, satisfies a normalization condition of the form (1.15). Therefore, by Remark 1.1 and conditions (1.15) and (1.16), we get

$$
M_{\alpha^{\prime}}\left(f\left(\Gamma_{k}^{\prime}\right)\right) \leqslant M_{\alpha^{\prime}}\left(f\left(\Gamma\left(\gamma_{k}, C_{1}, D\right)\right)\right) \leqslant \Delta(k),
$$


where $\Delta(k) \rightarrow 0$ as $k \rightarrow \infty$. However, $\Gamma_{k}=f\left(\Gamma_{k}^{\prime}\right)$. Therefore, using (2.6), we conclude that

$$
M_{\alpha^{\prime}}\left(\Gamma_{k}\right)=M_{\alpha^{\prime}}\left(f\left(\Gamma_{k}^{\prime}\right)\right) \leqslant \Delta(k) \rightarrow 0 \quad \text { as } k \rightarrow \infty .
$$

Relation (2.7), together with equality (2.5), contradicts inequality (2.4), which proves the possibility of continuous extension $f: \bar{D}_{P} \rightarrow \overline{D^{\prime}}$.

It remains to show that $f\left(\bar{D}_{P}\right)=\overline{D^{\prime}}$. It is clear, that $f\left(\bar{D}_{P}\right) \subset \overline{D^{\prime}}$. Now we show the inverse inclusion. Let $\zeta_{0} \in \overline{D^{\prime}}$. If $\zeta_{0} \in D^{\prime}$, then there exists $\xi_{0} \in D$ with $f\left(\xi_{0}\right)=\zeta_{0}$ and, consequently, $\zeta_{0} \in f(D)$. Assume that $\zeta_{0} \in \partial D^{\prime}$. Now there exists $\zeta_{m} \in D^{\prime}, \zeta_{m}=f\left(\xi_{m}\right), \xi_{m} \in D$, such that $\zeta_{m} \rightarrow \zeta_{0}$ as $m \rightarrow \infty$. By [ABBS, Theorem 10.10], $\bar{D}_{P}$ is a compact metric space. Now, we can consider that $\xi_{m} \rightarrow P_{0}$ as $m \rightarrow \infty$, where $P_{0}$ is some prime end in $\bar{D}_{P}$. Now $\zeta_{0} \in f\left(\bar{D}_{P}\right)$. The inclusion $\overline{D^{\prime}} \subset f\left(\bar{D}_{P}\right)$ has been proved. Consequently, $f\left(\bar{D}_{P}\right)=\overline{D^{\prime}}$. Lemma is proved.

\section{Proof of the main result}

We will say that a space $(X, d, \mu)$ is upper $\alpha$-regular at a point $x_{0} \in X$ if there is a constant $C>0$ such that

$$
\mu\left(B\left(x_{0}, r\right)\right) \leqslant C r^{\alpha}
$$

for the balls $B\left(x_{0}, r\right)$ centered at $x_{0} \in X$ with all radii $r<r_{0}$ for some $r_{0}>0$. We will also say that a space $(X, d, \mu)$ is upper $\alpha$-regular if the above condition holds at every point $x_{0} \in X$. The following statement can be found in [RS, Lemma 4.1].

Proposition 3.1. Let $G$ be a domain in Ahlfors upper $\alpha$-regular metric space $(X, d, \mu), \alpha \geqslant 2$. Assume that $x_{0} \in \bar{G}$ and $Q: G \rightarrow[0, \infty]$ belongs to $F M O\left(x_{0}\right)$. If

$$
\mu\left(G \cap B\left(x_{0}, 2 r\right)\right) \leqslant \gamma \cdot \log ^{\alpha-2} \frac{1}{r} \cdot \mu\left(G \cap B\left(x_{0}, r\right)\right)
$$

for some $r_{0}>0$ and every $r \in\left(0, r_{0}\right)$, then $Q$ satisfies (1.16) at $x_{0}$ with $\psi(t):=\frac{1}{t \log \frac{1}{t}}$.

Proof of the Theorem 1.1 follows from Lemma 2.1 and Proposition 3.1. Indeed, $X$ is upper regular by (1.1), and (3.1) holds because the measure is doubling by assumptions. So, the desired statement follows from the Lemma 2.1.

\section{Homeomorphic extension to the boundary}

Now we prove results about homeomorphic extension of mappings to the boundary in terms of prime ends. Let us give the following definition (see [MRSY, section 13.3], cf. [Va, Definition 17.5(4)] and [ $\mathrm{Na}_{2}$, Definition 2.8]). Let $(X, d, \mu)$ be metric space with finite Hausdorff dimension $\alpha \geqslant 1$. We say that the boundary of $D$ is weakly flat at a point $x_{0} \in \partial D$ if, for every number $P>0$ and every neighborhood $U$ of the point $x_{0}$, there is a neighborhood $V \subset U$ such that $M_{\alpha}(\Gamma(E, F, D)) \geqslant P$ for all continua $E$ and $F$ in $D$ intersecting $\partial U$ and $\partial V$. We say that the boundary $\partial D$ is weakly flat if the corresponding property holds at every point of the boundary. Given $P \in E_{D}$ and $f: D \rightarrow X^{\prime}$, set

$$
L=C(f, P):=\left\{y \in X^{\prime}: y=\lim _{k \rightarrow \infty} f\left(x_{k}\right), x_{k} \rightarrow P, x_{k} \in D\right\} .
$$

Analog of the following lemma was proved in [MRSY, Lemma 13.4] (see also [KR, Lemma 4] and [Sm, Lemma 5]).

Lemma 4.1. Let $D$ and $D^{\prime}$ be domains with finite Hausdorff dimensions $\alpha$ and $\alpha^{\prime} \geqslant 2$ in spaces $(X, d, \mu)$ and $\left(X^{\prime}, d^{\prime}, \mu^{\prime}\right)$, respectively. Assume that $X$ is complete 
and supports an $\alpha$-Poincaré inequality, and that the measure is doubling. Let $D$ be a bounded domain which is finitely connected at the boundary, and let $Q: X \rightarrow(0, \infty)$ be integrable function in $D, Q(x) \equiv 0$ for $x \in X \backslash D$. Suppose that $f: D \rightarrow D^{\prime}$, $D^{\prime}=f(D)$, is a ring $Q$-homeomorphism in $\partial D$, moreover, suppose that $\partial D^{\prime}$ is weakly flat and $\overline{D^{\prime}}$ is compact in $X^{\prime}$. If $P_{1}$ and $P_{2}$ are different prime ends in $E_{D}$, then $C\left(f, P_{1}\right) \cap C\left(f, P_{2}\right)=\varnothing$.

Proof. Assume that $C_{1} \cap C_{2} \neq \varnothing$, where $C_{i}=C\left(f, P_{i}\right), i=1,2$. Now, there exists $y_{0} \in C_{1} \cap C_{2}$.

I. Let $P_{1}=\left[E_{k}\right], k=1,2, \ldots$, and $P_{2}=\left[G_{l}\right], l=1,2, \ldots$, . By Remark 4.5 in [ABBS] we can consider that the sets $E_{k}$ and $G_{l}$ are open. By Remark 2.6 in [ABBS] the sets $E_{k}$ and $G_{l}$ is path connected for every $k, l \in \mathbf{N}$.

Let us show that there exists $k_{0} \in \mathbf{N}$ such that

$$
E_{k} \cap G_{k}=\varnothing \quad \forall k \geqslant k_{0} .
$$

Suppose the contrary, i.e., suppose that for every $l=1,2, \ldots$ there exists an increasing sequence $k_{l}, l=1,2, \ldots$, such that $x_{k_{l}} \in E_{k_{l}} \cap G_{k_{l}}, l=1,2, \ldots$ Now $x_{k_{l}} \rightarrow P_{1}$ and $x_{k_{l}} \rightarrow P_{2}, l \rightarrow \infty$. Let $m_{P}$ be the metric on $\bar{D}_{P}$ defined in Proposition 1.2. By triangle inequality,

$$
m_{P}\left(P_{1}, P_{2}\right) \leqslant m_{P}\left(P_{1}, x_{k_{l}}\right)+m_{P}\left(x_{k_{l}}, P_{2}\right) \rightarrow 0, \quad l \rightarrow \infty,
$$

that contradicts to Proposition 1.2. Thus, (4.1) holds, as required.

II. Denote $x_{0}:=I\left(\left[E_{k}\right]\right.$ ) (see Proposition 1.1). Arguing as in the proof of Lemma 2.1, we can show that, for every $r>0$ there exists $N \in \mathbf{N}$ such that

$$
E_{k} \subset B\left(x_{0}, r\right) \cap D \quad \forall k \geqslant N .
$$

Since $D$ is connected and $E_{k_{0}+1} \neq D$, we obtain that $\partial E_{k_{0}+1} \cap D \neq \varnothing$ (see [Ku, Ch. $5, \S 46$, item I]). Set $r_{0}:=d\left(x_{0}, \partial E_{k_{0}+1} \cap D\right)$. Since $\overline{E_{k_{0}}}$ is compact, $r_{0}>0$. By (4.2), there exists $m_{0} \in \mathbf{N}, m_{0}>k_{0}+1$, such that

$$
E_{k} \subset B\left(x_{0}, r_{0} / 2\right) \cap D \quad \forall k \geqslant m_{0} .
$$

III. Set $D_{0}:=E_{m_{0}+1}, D_{*}:=G_{m_{0}+1}$. Let us to show that

$$
\Gamma\left(D_{0}, D_{*}, D\right)>\Gamma\left(S\left(x_{0}, r_{0} / 2\right), S\left(x_{0}, r_{0}\right), A\left(x_{0}, r_{0} / 2, r_{0}\right)\right),
$$

where $A\left(x_{0}, r_{1}, r_{2}\right)$ is defined in (1.4). Assume that $\gamma \in \Gamma\left(D_{0}, D_{*}, D\right), \gamma:[0,1] \rightarrow D$. Set

$$
|\gamma|:=\{x \in D: \exists t \in[0,1]: \gamma(t)=x\}
$$

By (4.1), $|\gamma| \cap E_{k_{0}+1} \neq \varnothing \neq|\gamma| \cap\left(D \backslash E_{k_{0}+1}\right)$. Thus,

$$
|\gamma| \cap \partial E_{k_{0}+1} \neq \varnothing
$$

(see $[\mathrm{Ku}$, Theorem $1, \S 46$, item I]). Moreover, observe that

$$
\gamma(1) \notin \partial E_{k_{0}+1} \text {. }
$$

Suppose the contrary, i.e., that $\gamma(1) \in \partial E_{k_{0}+1}$. By definition of prime end, $\partial E_{k_{0}+1} \cap$ $D \subset \overline{E_{k_{0}}}$. Since $\operatorname{dist}\left(D \cap \partial E_{k+1}, D \cap \partial E_{k}\right)>0$ for all $k=1,2, \ldots$, we obtain that $\partial E_{k_{0}+1} \cap D \subset E_{k_{0}}$. Now, we have that $\gamma(1) \in E_{k_{0}}$ and, simultaneously, $\gamma(1) \in$ $G_{m_{0}+1} \subset G_{k_{0}}$. The last relations contradict with (4.1). Thus, (4.6) holds, as required.

By (4.3), we obtain that $|\gamma| \cap B\left(x_{0}, r_{0} / 2\right) \neq \varnothing$. We prove that $|\gamma| \cap(D \backslash$ $\left.B\left(x_{0}, r_{0} / 2\right)\right) \neq \varnothing$. In fact, if it is not true, then $\gamma(t) \in B\left(x_{0}, r_{0} / 2\right)$ for every $t \in[0,1]$. However, by (4.5) we obtain that $\left(\partial E_{k_{0}+1} \cap D\right) \cap B\left(x_{0}, r_{0} / 2\right) \neq \varnothing$, that contradicts to the definition of $r_{0}$. Thus, $|\gamma| \cap\left(D \backslash B\left(x_{0}, r_{0} / 2\right)\right) \neq \varnothing$, as required. Now, 
by $\left[\mathrm{Ku}\right.$, Theorem $1, \S 46$, item I], there exists $t_{1} \in(0,1]$ with $\gamma\left(t_{1}\right) \in S\left(x_{0}, r_{0} / 2\right)$. We can consider that $t_{1}=\max \left\{t \in[0,1]: \gamma(t) \in S\left(x_{0}, r_{0} / 2\right)\right\}$. We prove that $t_{1} \neq 1$. Suppose the contrary, i.e., suppose that $t_{1}=1$. Now, we obtain that $\gamma(t) \in B\left(x_{0}, r_{0} / 2\right)$ for every $t \in[0,1)$. On the other hand, by (4.5) and (4.6), we obtain that $\partial E_{k_{0}+1} \cap B\left(x_{0}, r_{0} / 2\right) \neq \varnothing$, which contradicts to the definition of $r_{0}$. Thus, $t_{1} \neq 1$, as required. Set $\gamma_{1}:=\left.\gamma\right|_{\left[t_{1}, 1\right]}$.

By the definition, $\left|\gamma_{1}\right| \cap B\left(x_{0}, r_{0}\right) \neq \varnothing$. We prove that $\left|\gamma_{1}\right| \cap\left(D \backslash B\left(x_{0}, r_{0}\right)\right) \neq \varnothing$. In fact, assume the contrary, i.e., assume that $\gamma_{1}(t) \in B\left(x_{0}, r_{0}\right)$ for every $t \in\left[t_{1}, 1\right]$. Since $\gamma(t) \in B\left(x_{0}, r_{0} / 2\right)$ for $t<t_{1}$, by (4.5) we obtain that $\left|\gamma_{1}\right| \cap \partial E_{k_{0}+1} \neq \varnothing$. Consequently, $B\left(x_{0}, r_{0}\right) \cap\left(\partial E_{k_{0}+1} \cap D\right) \neq \varnothing$, that contradicts to the definition of $r_{0}$. Thus, $\left|\gamma_{1}\right| \cap\left(D \backslash B\left(x_{0}, r_{0}\right)\right) \neq \varnothing$, as required. Now, by [Ku, Theorem $1, \S 46$, item I], there exists $t_{2} \in\left(t_{1}, 1\right]$ with $\gamma\left(t_{2}\right) \in S\left(x_{0}, r\right)$. We can consider that $t_{2}=$ $\min \left\{t \in\left[t_{1}, 1\right]: \gamma(t) \in S\left(x_{0}, r_{0}\right)\right\}$. We put $\gamma_{2}:=\left.\gamma\right|_{\left[t_{1}, t_{2}\right]}$. Observe that $\gamma>\gamma_{2}$ and $\gamma_{2} \in \Gamma\left(S\left(x_{0}, r_{0} / 2\right), S\left(x_{0}, r_{0}\right), A\left(x_{0}, r_{0} / 2, r_{0}\right)\right)$. Thus, (4.4) has been proved.

IV. Consider the function

$$
\eta(t)= \begin{cases}2 / r_{0}, & t \in\left(r_{0} / 2, r_{0}\right) \\ 0, & t \in \mathbf{R} \backslash\left(r_{0} / 2, r_{0}\right) .\end{cases}
$$

Note that $\eta$ satisfies (1.6) with $r_{1}:=r_{0} / 2$ and $r_{2}:=r_{0}$. Set $S_{1}:=S\left(x_{0}, r_{0} / 2\right)$, $S_{2}:=S\left(x_{0}, r_{0}\right), A:=A\left(x_{0}, r_{0} / 2, r_{0}\right)$. Thus, by (1.2), (1.5) and (4.4), we obtain that

$$
M_{\alpha^{\prime}}\left(f\left(\Gamma\left(D_{0}, D_{*}, D\right)\right)\right) \leqslant M_{\alpha^{\prime}}\left(f\left(\Gamma\left(S_{1}, S_{2}, A\right)\right)\right) \leqslant\left(\frac{2}{r_{0}}\right)^{\alpha} \cdot\|Q\|_{L^{1}(D)}<\infty .
$$

Set $M_{0}:=\left(\frac{2}{r_{0}}\right)^{\alpha} \cdot\|Q\|_{L^{1}(D)}, 0<M_{0}<\infty$. Now, by (4.7) we obtain that

$$
M_{\alpha^{\prime}}\left(f\left(\Gamma\left(D_{0}, D_{*}, D\right)\right)\right) \leqslant M_{0}
$$

V. Let us to show that there exists $l_{0}>0$ such that

$$
S\left(y_{0}, l_{0}\right) \cap f\left(D_{0}\right) \neq \varnothing, \quad S\left(y_{0}, l_{0}\right) \cap f\left(D_{*}\right) \neq \varnothing .
$$

In fact, since $y_{0} \in C_{1} \cap C_{2}$, we obtain that $y_{0} \in \overline{f\left(D_{0}\right)}$. Now, given $r_{1}>0$, there exists $x_{1} \in B\left(y_{0}, r_{1}\right) \cap f\left(D_{0}\right)$. Similarly, $y_{0} \in \overline{f\left(D_{*}\right)}$, and there exists $x_{2} \in B\left(y_{0}, r_{1}\right) \cap f\left(D_{*}\right)$. Set $l_{0}:=\min \left\{d^{\prime}\left(y_{0}, x_{1}\right), d^{\prime}\left(y_{0}, x_{2}\right)\right\}$. We have that $f\left(D_{0}\right) \cap B\left(y_{0}, l_{0}\right) \neq \varnothing \neq f\left(D_{0}\right) \backslash$ $B\left(y_{0}, l_{0}\right)$ and $f\left(D_{*}\right) \cap B\left(y_{0}, l_{0}\right) \neq \varnothing \neq f\left(D_{*}\right) \backslash B\left(y_{0}, l_{0}\right)$. By [Ku, Theorem $1, \S 46$, item I] we obtain (4.9), as required.

Since $\partial D^{\prime}$ is weakly flat, there exists $r_{*} \in\left(0, l_{0}\right)$ such that

$$
M_{\alpha^{\prime}}\left(\Gamma\left(E, F, D^{\prime}\right)\right)>M_{0}
$$

for each continua $E$ and $F$ in $D^{\prime}$ such that $E \cap S\left(y_{0}, l_{0}\right) \neq \varnothing \neq E \cap S\left(y_{0}, r_{*}\right)$ and $F \cap S\left(y_{0}, l_{0}\right) \neq \varnothing \neq F \cap S\left(y_{0}, r_{*}\right)$. By (4.9) there exist curves $c_{1}$ and $c_{2}$, which join $S\left(y_{0}, l_{0}\right)$ and $S\left(y_{0}, r_{*}\right)$ in domains $f\left(D_{0}\right)$ and $f\left(D_{*}\right)$, correspondingly. Put $E:=c_{1}$ and $F:=c_{2}$. Observe that $\Gamma\left(c_{1}, c_{2}, D^{\prime}\right) \subset f\left(\Gamma\left(D_{0}, D_{*}, D\right)\right)$. Now, by (4.10) we obtain that

$$
M_{0}<M_{\alpha^{\prime}}\left(\Gamma\left(c_{1}, c_{2}, D^{\prime}\right)\right) \leqslant M_{\alpha^{\prime}}\left(f\left(\Gamma\left(D_{0}, D_{*}, D\right)\right)\right),
$$

that contradicts (4.8). Thus, $C\left(f, P_{1}\right) \cap C\left(f, P_{2}\right)=\varnothing$, as required.

There are two important statements which follow from Lemma 4.1.

Theorem 4.1. Under conditions of Lemma 4.1, $f^{-1}$ has a continuous extension $\overline{f^{-1}}: \bar{D}^{\prime} \rightarrow \bar{D}_{P}$ such that $\overline{f^{-1}}\left(\overline{D^{\prime}}\right)=\bar{D}_{P}$. 
Proof. Let us to show that, given $\zeta_{0} \in \partial D^{\prime}$, a set $C\left(f^{-1}, \zeta_{0}\right)$ is a single point $\xi_{0} \in E_{D}$, where $E_{D}$ denotes prime ends space of $D$. In fact, assume that $x_{k} \stackrel{d^{\prime}}{\rightarrow} \zeta_{0}$ as $k \rightarrow \infty$ and $y_{k} \stackrel{d^{\prime}}{\rightarrow} \zeta_{0}$ as $k \rightarrow \infty$. By [ABBS, Theorem 10.10], $\bar{D}_{P}$ is a compact metric space. Thus, we can consider that $f^{-1}\left(x_{k}\right) \rightarrow P_{1} \in E_{D}$ and $f^{-1}\left(y_{k}\right) \rightarrow P_{2} \in E_{D}$ as $k \rightarrow \infty$. If $P_{1} \neq P_{2}$, then $\zeta_{0} \in C\left(f, P_{1}\right) \cap C\left(f, P_{2}\right)$ that contradicts to the lemma 4.1.

Thus, we have the extension $\overline{f^{-1}}$ of $f^{-1}$ on $\overline{D^{\prime}}$ such that $C\left(f^{-1}, \partial D^{\prime}\right) \subset \bar{D}_{P} \backslash D$. Let us to show that $C\left(\overline{f-1}, \partial D^{\prime}\right)=\bar{D}_{P} \backslash D$. Given $P_{0} \in E_{D}$, we can find $x_{m} \rightarrow P_{0}$ as $m \rightarrow \infty$. Since $\mu$ is doubling, $X$ is complete if and only if it is proper (i.e. every closed bounded set is compact), see [BB, Proposition 3.1]. Since $D$ is bounded, $\bar{D}$ is compact. By assumptions of the theorem, $\overline{D^{\prime}}$ is compact, as well. Thus, we may assume that $x_{m} \rightarrow x_{0} \in \partial D$ and $f\left(x_{m}\right) \stackrel{d^{\prime}}{\rightarrow} \zeta_{0} \in \partial D^{\prime}$ and $m \rightarrow \infty$. Thus, $P_{0} \in C\left(f^{-1}, \zeta_{0}\right)$, as required.

Finally, let us to show that $\overline{f^{-1}}: \overline{D^{\prime}} \rightarrow \bar{D}_{P}$ is continuous in $\overline{D^{\prime}}$. In fact, assume that $\zeta_{m} \rightarrow \zeta_{0}$ as $m \rightarrow \infty, \zeta_{m}, \zeta_{0} \in \overline{D^{\prime}}$. If $\zeta_{0} \in D^{\prime}$, the desired conclusion is obvious. Now, assume that $\zeta_{0} \in \partial D^{\prime}$. We choose $\zeta_{m}^{*} \in D^{\prime}$ such that $d^{\prime}\left(\zeta_{m}, \zeta_{m}^{*}\right)<1 / m$ and $m_{P}\left(\overline{f^{-1}}\left(\zeta_{m}\right), \overline{f^{-1}}\left(\zeta_{m}^{*}\right)\right)<1 / m$, where $m_{P}$ is the metric defined in Proposition 1.2. Since $\zeta_{m}^{*} \stackrel{d^{\prime}}{\rightarrow} \zeta_{0}$, we obtain that $\overline{f^{-1}}\left(\zeta_{m}^{*}\right) \rightarrow \overline{f^{-1}}\left(\zeta_{0}\right)$ as $m \rightarrow \infty$. Thus, $\overline{f^{-1}}\left(\zeta_{m}\right) \rightarrow$ $\overline{f^{-1}}\left(\zeta_{0}\right)$, as required.

Example. Given $n \geqslant 2, p \geqslant 1$ and $\alpha \in(0, n / p(n-1))$, set

$$
f(x)=\frac{1+|x|^{\alpha}}{|x|} \cdot x, \quad x \in \mathbf{B}^{n} \backslash\{0\} .
$$

It is not difficult to see that $f$ is a ring $Q$-homeomorphism of $\mathbf{B}^{n} \backslash\{0\}$ onto $A:=$ $\{1<|y|<2\}$, where $Q(x):=\left(\frac{1+r^{\alpha}}{\alpha r^{\alpha}}\right)^{n-1}, r=|x|$ (see, e.g., [MRSY, Proposition 6.3]). Moreover, $Q \in L^{p}\left(\mathbf{B}^{n}\right)$.

Since $\partial A$ consists of two spheres $\mathbf{S}^{n-1}$ and $S(0,2)$, which are $C^{1}$-manifolds, $A$ is a collared domain, i.e., each boundary point of the domain has an arbitrarily small neighborhood such that the part of the neighborhood inside the domain is quasiconformally equivalent to a ball, see [ $\mathrm{Na}_{4}$, Section 2.2], cf. [Va, Theorem 17.12] and $\left[\mathrm{Na}_{1}\right.$, Remark 1.5]. Thus, we may consider that all prime ends in $A$ are single points of $\partial A$ (see $\left[\mathrm{Na}_{4}\right.$, Theorem 4.1]). Observe that $f$ has no continuous extension at 0 , however, the inverse mapping $f^{-1}(y)=\frac{y}{|y|}(|y|-1)^{1 / \alpha}$ is continuous in $\bar{A}$. In particular, $f^{-1}\left(\mathbf{S}^{n-1}\right)=0$. Thus, the statement of the Theorem 4.1 is not valid for $f$, but is valid for $f^{-1}$. In this case, $Q \notin F M O(0)$.

Combining Theorem 1.1 with Lemma 4.1, we obtain the following statement.

Theorem 4.2. Let $D$ and $D^{\prime}$ be domains with finite Hausdorff dimensions $\alpha$ and $\alpha^{\prime} \geqslant 2$ in spaces $(X, d, \mu)$ and $\left(X^{\prime}, d^{\prime}, \mu^{\prime}\right)$, respectively. Assume that $X$ is complete and supports an $\alpha$-Poincaré inequality, and that the measure is doubling. Let $D$ be a bounded domain which is finitely connected at the boundary, and let $Q: X \rightarrow(0, \infty)$ be an integrable function in $D$. Suppose that $f: D \rightarrow D^{\prime}, D^{\prime}=f(D)$, is a ring $Q$ homeomorphism in $\partial D$. Moreover, suppose that $\partial D^{\prime}$ is weakly flat and $\overline{D^{\prime}}$ is compact in $X^{\prime}$. Then $f$ has a homeomorphic extension $f: \bar{D}_{P} \rightarrow{\overline{D^{\prime}}}^{\prime}, f\left(\bar{D}_{P}\right)=\overline{D^{\prime}}$, whenever $Q \in F M O(\partial D)$. 


\section{Equicontinuity of families of homeomorphisms}

Now we prove that the corresponding families of ring $Q$-homeomorphisms are equicontinuous in $\bar{D}_{P}=D \cup E_{D}$, where $E_{D}$ is a prime ends space. In this section, we restrict us by a case of homeomorphisms, only. Let us recall some definitions. Let $(X, d)$ and $\left(X^{\prime}, d^{\prime}\right)$ be metric spaces with distances $d$ and $d^{\prime}$, respectively. A family $\mathfrak{F}$ of mappings $f: X \rightarrow X^{\prime}$ is said to be equicontinuous at a point $x_{0} \in X$ if for every $\varepsilon>0$ there is $\delta>0$ such that $d^{\prime}\left(f(x), f\left(x_{0}\right)\right)<\varepsilon$ for all $f \in \mathfrak{F}$ and $x \in X$ with $d\left(x, x_{0}\right)<\delta$. The family $\mathfrak{F}$ is equicontinuous if $\mathfrak{F}$ is equicontinuous at every point $x_{0} \in X$. In what follows, $X=\bar{D}_{P}$ and $d=m_{P}$, where $m_{P}$ is defined in Proposition 1.2. The next definition can be found, e.g., in [NP]. A domain $D$ is called a uniform domain if, for each $r>0$, there is $\delta>0$ such that $M_{\alpha}\left(\Gamma\left(F, F^{*}, D\right)\right) \geqslant \delta$ whenever $F$ and $F^{*}$ are continua of $D$ with $d(F) \geqslant r$ and $d\left(F^{*}\right) \geqslant r$. Domains $D_{i}, i \in I$, are said to be equi-uniform domains if, for $r>0$, the modulus condition above is satisfied by each $D_{i}$ with the same number $\delta$. It should be noted that the proposed concept of a uniform domain has, generally speaking, no relation to definition, introduced in [MS].

Given $\delta>0, D \subset X$ and a measurable function $Q: D \rightarrow[0, \infty]$, denote $\mathfrak{R}_{Q, \delta}(D)$ the family of all ring $Q$-homeomorphisms $f: D \rightarrow X^{\prime} \backslash K_{f}$ in $D$, such that $f(D)$ is some open set in $X^{\prime}$ and $d^{\prime}\left(K_{f}\right)=\sup _{x, y \in K_{f}} d^{\prime}(x, y) \geqslant \delta$, where $K_{f} \subset X^{\prime}$ is a continuum. The following statement holds.

Lemma 5.1. Let $(X, d, \mu)$ and $\left(X^{\prime}, d^{\prime}, \mu^{\prime}\right)$ be metric spaces, let $D$ be a domain in $X$ with finite Hausdorff dimension $\alpha \geqslant 2$, and let $X^{\prime}$ be a domain with finite Hausdorff dimension $\alpha^{\prime} \geqslant 2$. Given $x_{0} \in D$, assume that conditions (1.15)-(1.16) hold. If $X$ is locally path connected and locally compact space, and $X^{\prime}$ is a uniform domain, then $\mathfrak{R}_{Q, \delta}(D)$ is equicontinuous at $x_{0}$.

Proof. The idea of a proof is closely related to $\left[\mathrm{Sev}_{2}\right.$, Lemma 2]. Assume the contrary, i.e., assume that $\mathfrak{R}_{Q, \delta}(D)$ is not equicontinuous at $x_{0}$. Now, there is exists $x_{k} \in D$ and $f_{k} \in \mathfrak{R}_{Q, \delta}(D)$ such that $x_{k} \rightarrow x_{0}$ as $k \rightarrow \infty$ and

$$
d^{\prime}\left(f_{k}\left(x_{k}\right), f_{k}\left(x_{0}\right)\right) \geqslant \varepsilon_{0}
$$

for some $\varepsilon_{0}$. Since $X$ is locally connected by assumption, there is a sequence of balls $B\left(x_{0}, \varepsilon_{k}\right), k=0,1,2, \ldots, \varepsilon_{k} \rightarrow 0$ as $k \rightarrow \infty$, such that $V_{k+1} \subset \overline{B\left(x_{0}, \varepsilon_{k}\right)} \subset V_{k}$, where the $V_{k}$ are continua in $D$. There is no loss of generality in assuming that $x_{k} \in V_{k}$. Now, $x_{0}$ and $x_{k}$ can be joined by a curve $\gamma_{k}$ in the domain $V_{k}$. Note that an arbitrary curve $\gamma \in \Gamma\left(K_{f_{k}}, f_{k}\left(\gamma_{k}\right), X^{\prime}\right)$ is not included entirely both in $f_{k}\left(B\left(x_{0}, \varepsilon_{0}\right)\right)$ and $X^{\prime} \backslash f_{k}\left(B\left(x_{0}, \varepsilon_{0}\right)\right.$ ), therefore there exists $y_{1} \in|\gamma| \cap f_{k}\left(S\left(x_{0}, \varepsilon_{0}\right)\right)$ (see $[\mathrm{Ku}$, Theorem $1, \S 46$, item I]). Let $\gamma:[0,1] \rightarrow X^{\prime}$ and let $t_{1} \in(0,1)$ be such that $\gamma\left(t_{1}\right)=y_{1}$. There is no loss of generality in assuming that $|\gamma|_{\left[0, t_{1}\right)} \mid \subset f_{k}\left(B\left(x_{0}, \varepsilon_{0}\right)\right)$. We put $\gamma_{1}:=\left.\gamma\right|_{\left[0, t_{1}\right)}$, and $\alpha_{1}=f_{k}^{-1}\left(\gamma_{1}\right)$. Observe that $\left|\alpha_{1}\right| \subset B\left(x_{0}, \varepsilon_{0}\right)$, moreover, $\alpha_{1}$ is not included entirely either in $\overline{B\left(x_{0}, \varepsilon_{k-1}\right)}$, or in $X \backslash \overline{B\left(x_{0}, \varepsilon_{k-1}\right)}$. Consequently, there exists $t_{2} \in\left(0, t_{1}\right)$ with $\alpha_{1}\left(t_{2}\right) \in S\left(x_{0}, \varepsilon_{k-1}\right)$ (see $[\mathrm{Ku}$, Theorem $1, \S 46$, item I]). There is no loss of generality in assuming that $\left|\alpha_{1}\right|_{\left[t_{2}, t_{1}\right]} \mid \subset X \backslash \overline{B\left(x_{0}, \varepsilon_{k-1}\right)}$. Put $\alpha_{2}=\left.\alpha_{1}\right|_{\left[t_{2}, t_{1}\right]}$. Observe that $\gamma_{2}:=f_{k}\left(\alpha_{2}\right)$ is a subcurve of $\gamma$. By the said above,

$$
\Gamma\left(K_{f_{k}}, f_{k}\left(\gamma_{k}\right), X^{\prime}\right)>\Gamma\left(f_{k}\left(S\left(x_{0}, \varepsilon_{k-1}\right)\right), f_{k}\left(S\left(x_{0}, \varepsilon_{0}\right)\right), f_{k}(A)\right),
$$

where $A=A\left(x_{0}, \varepsilon_{k-1}, \varepsilon_{0}\right)$, and by (1.2) we obtain

$$
M_{\alpha^{\prime}}\left(\Gamma\left(K_{f_{k}}, f_{k}\left(\gamma_{k}\right), X^{\prime}\right)\right) \leqslant M_{\alpha^{\prime}}\left(\Gamma\left(f_{k}\left(S\left(x_{0}, \varepsilon_{k-1}\right)\right), f_{k}\left(S\left(x_{0}, \varepsilon_{0}\right)\right), f_{k}(A)\right)\right) .
$$


Since $I\left(\varepsilon, \varepsilon_{0}\right) \rightarrow \infty$ as $\varepsilon \rightarrow 0$, we can consider that $I\left(\varepsilon_{k}, \varepsilon_{0}\right)>0$ for every $k=1,2, \ldots$ Consider the family of measurable functions

$$
\eta_{k}(t)=\psi(t) / I\left(\varepsilon_{k}, \varepsilon_{0}\right), \quad t \in\left(\varepsilon, \varepsilon_{0}\right) .
$$

Observe that $\int_{\varepsilon_{k}}^{\varepsilon_{0}} \eta_{k}(t) d t=1$. Now, by (1.3), (1.16) and (5.2), we obtain that

$$
M_{\alpha^{\prime}}\left(\Gamma\left(K_{f_{k}}, f_{k}\left(\gamma_{k}\right), X^{\prime}\right)\right) \leqslant \varphi\left(\varepsilon_{k}\right),
$$

where $\varphi$ is some function with $\varphi\left(\varepsilon_{k}\right) \rightarrow 0$ as $k \rightarrow \infty$. On the other hand, it follows from (5.1) that $\min \left\{d^{\prime}\left(K_{f_{k}}\right), d^{\prime}\left(f_{k}\left(\gamma_{k}\right)\right)\right\} \geqslant r_{0}$ for some $r_{0}>0$ and every $k=1,2, \ldots$, . Now, since $X^{\prime}$ is uniformly domain, we obtain that

$$
M_{\alpha^{\prime}}\left(\Gamma\left(K_{f_{k}}, f_{k}\left(\gamma_{k}\right), X^{\prime}\right)\right) \geqslant \delta_{0}
$$

for some $\delta_{0}>0$ and every $k=1,2, \ldots$, . Now, (5.4) contradicts with (5.3). Thus, $\mathfrak{R}_{Q, \delta}(D)$ is equicontinuous at $x_{0}$, as required.

Given $\delta>0, D \subset X$, a continuum $A \subset D$ and a measurable function $Q: D \rightarrow$ $[0, \infty]$, denote $\mathfrak{F}_{Q, \delta, A}(D)$ the family of all ring $Q$-homeomorphisms $f: D \rightarrow X^{\prime} \backslash K_{f}$ in $\bar{D}$, such that $f(D)$ is some open set in $X^{\prime}$ and $d^{\prime}\left(K_{f}\right)=\sup _{x, y \in K_{f}} d^{\prime}(x, y) \geqslant \delta$ and $d^{\prime}(f(A)) \geqslant \delta$, where $K_{f} \subset X^{\prime}$ is a continuum. An analog of a following result was proved in [NP, Theorem 3.1].

Lemma 5.2. Let $D$ and $D_{f}^{\prime}:=f(D), f \in \mathfrak{F}_{Q, \delta, A}(D)$, be domains with finite Hausdorff dimensions $\alpha$ and $\alpha^{\prime} \geqslant 2$ in spaces $(X, d, \mu)$ and $\left(X^{\prime}, d^{\prime}, \mu^{\prime}\right)$, respectively, and let $X^{\prime}$ be a domain with finite Hausdorff dimension $\alpha^{\prime} \geqslant 2$. Assume that $X$ is complete and supports an $\alpha$-Poincaré inequality, and that the measure is doubling. Let $D$ be a bounded domain which is finitely connected at the boundary, and let $Q: X \rightarrow(0, \infty)$ be a locally integrable function. Assume that, for every $x_{0} \in \bar{D}$ conditions (1.15)-(1.16) hold. If $D_{f}^{\prime}:=f(D)$ and $X^{\prime}$ are equi-uniform domains over $f \in \mathfrak{F}_{Q, \delta, A}(D)$ and $\overline{D_{f}^{\prime}}$ are compacts in $X^{\prime}$, then every $f \in \mathfrak{F}_{Q, \delta, A}(D)$ has a continuous extension $f: \bar{D}_{P} \rightarrow \overline{D_{f}^{\prime}}$, and $\mathfrak{F}_{Q, \delta, A}(D)$ is equicontinuous in $\bar{D}_{P}$.

Proof. Observe that $\partial D_{f}^{\prime}=\partial f(D)$ is strongly accessible for every $f \in \mathfrak{F}_{Q, \delta, A}(D)$. Indeed, assume that $x_{0} \in \partial D_{f}^{\prime}$. Given a neighborhood $U$ of $x_{0}$, there exists $\varepsilon_{1}>0$ such that $V:=B\left(x_{0}, \varepsilon_{1}\right), \overline{B\left(x_{0}, \varepsilon_{1}\right)} \subset U$. Assume that $\partial U \neq \varnothing$ and $\partial V \neq \varnothing$, now $\varepsilon_{2}:=d^{\prime}(\partial U, \partial V)>0$. Since $D_{f}^{\prime}$ are equi-uniform, we obtain that $M_{\alpha^{\prime}}\left(\Gamma\left(F, G, D_{f}^{\prime}\right)\right) \geqslant$ $\delta$ for some $\delta>0$, because $d^{\prime}(F) \geqslant \varepsilon_{2}$ and $d^{\prime}(G) \geqslant \varepsilon_{2}$, whenever $F$ and $G$ are continua in $D_{f}^{\prime}$ with $F \cap \partial U \neq \varnothing \neq F \cap \partial V$ and $G \cap \partial U \neq \varnothing \neq G \cap \partial V$. Thus, $\partial D_{f}^{\prime}=\partial f(D)$ is strongly accessible, as required. Now, by Lemma 2.1 every $f \in \mathfrak{F}_{Q, \delta, A}(D)$ has a continuous extension $f: \bar{D}_{P} \rightarrow \overline{D_{f}^{\prime}}$.

Since $\mu$ is doubling, $X$ is complete if and only if it is proper (i.e. every closed bounded set is compact), see [BB, Proposition 3.1]. Now, $X$ is a locally compact space. Since $X$ is complete, $X$ supports an $\alpha$-Poincaré inequality, and the measure is doubling, we obtain that $X$ is locally connected (see [ABBS], see also [Ch, Theorem 17.1]). Moreover, $X$ is locally path connected by the Mazurkiewicz-MooreMenger theorem (see in $[\mathrm{Ku}$, Theorem 1, Ch. 6, § 50, item II]. Thus, all conditions of Lemma 5.1 are satisfied. Now, by Lemma $5.1, \mathfrak{F}_{Q, \delta, A}(D)$ is equicontinuous at $x_{0}$ for every $x_{0} \in D$.

It remains to show that $\mathfrak{F}_{Q, \delta, A}(D)$ is equicontinuous on $E_{D}=\bar{D}_{P} \backslash D$. Assume the contrary, i.e., assume that there exists $P_{0} \in E_{D}$ such that $\mathfrak{F}_{Q, \delta, A}(D)$ is not 
equicontinuous at $P_{0}$. Now, there is exists $P_{k} \in \bar{D}_{P}$ and $f_{k} \in \mathfrak{F}_{Q, \delta, A}(D)$ such that $P_{k} \rightarrow P_{0}$ as $k \rightarrow \infty$ and

$$
d^{\prime}\left(f_{k}\left(P_{k}\right), f_{k}\left(P_{0}\right)\right) \geqslant \varepsilon_{0}
$$

for some $\varepsilon_{0}$. Since $f_{k}$ has a continuous extension on $\bar{D}_{P}$, given $k \in \mathbf{N}$, we can find $x_{k} \in D$ with $m_{P}\left(x_{k}, P_{k}\right)<1 / k$ and $d\left(f_{k}\left(x_{k}\right), f_{k}\left(P_{k}\right)\right)<1 / k$. Thus, we obtain from (5.5) that

$$
d^{\prime}\left(f_{k}\left(x_{k}\right), f_{k}\left(P_{0}\right)\right) \geqslant \varepsilon_{0} / 2 \quad \forall k=1,2, \ldots
$$

Similarly, we can find $x_{k}^{\prime} \in D$ such that $x_{k}^{\prime} \rightarrow P_{0}$ as $k \rightarrow \infty$, and $d^{\prime}\left(f_{k}\left(x_{k}^{\prime}\right), f_{k}\left(P_{0}\right)\right)<$ $1 / k, k=1,2, \ldots$. Thus, we obtain from (5.6) that

$$
d^{\prime}\left(f_{k}\left(x_{k}\right), f_{k}\left(x_{k}^{\prime}\right)\right) \geqslant \varepsilon_{0} / 4 \quad \forall k=1,2, \ldots,
$$

where $x_{k}$ and $x_{k}^{\prime} \in D$ satisfy conditions $x_{k} \rightarrow P_{0}, x_{k}^{\prime} \rightarrow P_{0}$ as $k \rightarrow \infty$.

Denote $x_{0}:=I\left(\left[E_{k}\right]\right.$ ) (see Proposition 1.1). By Remark 4.5 in [ABBS] we can consider that the sets $E_{k}$ are open. Moreover, by Remark 2.6 in [ABBS] the set $E_{k}$ is path connected for every $k \in \mathbf{N}$. Arguing as in the proof of Lemma 2.1, we can show that, for every $r>0$ there exists $k \in \mathbf{N}$ such that $E_{k} \subset B\left(x_{0}, r\right) \cap D$. Thus, there is no loss of generality in assuming that $x_{k}, x_{k}^{\prime} \in E_{k}$ and $E_{k} \subset B\left(x_{0}, 2^{-k}\right)$. Let $\gamma_{k}$ be a path, joining $x_{k}$ and $x_{k}^{\prime}$ in $E_{k}$. Observe that $A \subset D \backslash B\left(x_{0}, 2^{-k}\right)$ for all $k>k_{0}$ and some $k_{0} \in \mathbf{N}$. We can consider that $2^{-k_{0}}<\varepsilon_{0}$. Let $\Gamma_{k}$ be a family of curves joining $\gamma_{k}$ and $A$ in $D_{f_{k}}^{\prime}$. By Remark 1.1, we obtain that

$$
M_{\alpha^{\prime}}\left(f_{k}\left(\Gamma_{k}\right)\right) \leqslant M_{\alpha^{\prime}}\left(f_{k}\left(\Gamma\left(S\left(x_{0}, 2^{-k}\right), S\left(x_{0}, 2^{-k_{0}}\right), A\left(x_{0}, 2^{-k}, 2^{-k_{0}}\right)\right)\right)\right) .
$$

Observe that

$$
\eta(t)= \begin{cases}\psi(t) / I\left(2^{-k}, 2^{-k_{0}}\right), & t \in\left(2^{-k}, 2^{-k_{0}}\right), \\ 0, & t \in \mathbf{R} \backslash\left(2^{-k}, 2^{-k_{0}}\right),\end{cases}
$$

$I\left(\varepsilon, \varepsilon_{0}\right):=\int_{\varepsilon}^{\varepsilon_{0}} \psi(t) d t$, satisfies the condition (1.6) at $r_{1}=2^{-k}$ and $r_{2}=2^{-k_{0}}$. By the definition of a ring $Q$-homeomorphism at a boundary point, (1.16) and (5.8) imply

$$
M_{p}\left(f_{k}\left(\Gamma_{k}\right)\right) \leqslant \alpha\left(2^{-k}\right) \rightarrow 0
$$

as $k \rightarrow \infty$, where $\alpha(\varepsilon)$ is some nonnegative function with $\alpha(\varepsilon) \rightarrow 0$ as $\varepsilon \rightarrow 0$.

However, $f_{k}\left(\Gamma_{k}\right)=\Gamma\left(f_{k}\left(\gamma_{k}\right), f_{k}(A), D_{f_{k}}^{\prime}\right)$. By assumption, $d^{\prime}\left(f_{k}(A)\right) \geqslant \delta, k=$ $1,2, \ldots$, moreover, by (5.7) we obtain that $d^{\prime}\left(f_{k}\left(\gamma_{k}\right)\right) \geqslant \varepsilon_{0} / 4, k=1,2, \ldots$. Since $D_{f_{k}}^{\prime}$ are are equi-uniform domains, we obtain that

$$
M_{\alpha^{\prime}}\left(f_{k}\left(\Gamma_{k}\right)\right) \geqslant r_{0}, k=1,2, \ldots,
$$

for some $r_{0}>0$. But (5.10) contradicts (5.9). Thus, $\mathfrak{F}_{Q, \delta, A}(D)$ is equicontinuous at $P_{0}$, as required.

The following main result holds.

Theorem 5.1. Let $D$ and $D_{f}^{\prime}:=f(D), f \in \mathfrak{F}_{Q, \delta, A}(D)$, be domains with finite Hausdorff dimensions $\alpha$ and $\alpha^{\prime} \geqslant 2$ in spaces $(X, d, \mu)$ and $\left(X^{\prime}, d^{\prime}, \mu^{\prime}\right)$, respectively, and let $X^{\prime}$ be a domain with finite Hausdorff dimension $\alpha^{\prime} \geqslant 2$. Assume that $X$ is complete and supports an $\alpha$-Poincaré inequality, and that the measure is doubling. Let $D$ be a bounded domain which is finitely connected at the boundary, and let $Q: X \rightarrow(0, \infty)$ be a locally integrable function. Assume that, $Q \in F M O(\bar{D})$. If $D_{f}^{\prime}:=f(D)$ and $X^{\prime}$ are equi-uniform domains over $f \in \mathfrak{F}_{Q, \delta, A}(D)$ and $\overline{D_{f}^{\prime}}$ are compacts in $X^{\prime}$, then $\mathfrak{F}_{Q, \delta, A}(D)$ is equicontinuous in $\bar{D}_{P}$. 
Proof of the Theorem 5.1 follows from Lemma 5.2 and Proposition 3.1. Indeed, $X$ is upper regular by (1.1), and (3.1) holds because the measure is doubling by assumptions. So, the desired statement follows from the Lemma 5.2.

Remark 5.1. By definition, $\mathfrak{F}_{Q, \delta, A}(D) \subset \mathfrak{R}_{Q, \delta}(D)$. The following example shows that this inclusion, generally speaking, is strict. For simplicity, let $X=X^{\prime}=\mathbf{C}, \mu=$ $\mu^{\prime}=m$, where $m$ is the Lebesgue measure in $\mathbf{C}$, and let $d(x, y)=d^{\prime}(x, y)=|x-y|$. As above, $\mathbf{D}$ denotes the unit disk in $\mathbf{C}$.

As known, the linear-fractional automorphisms of the unit disk $\mathbf{D} \subset \mathbf{C}$ onto itself can be written by the formula $f(z)=e^{i \theta} \frac{z-a}{1-\bar{a} z}, z \in \mathbf{D}, a \in \mathbf{C},|a|<1, \theta \in[0,2 \pi)$. These mappings $f$ are ring 1 -homeomorphisms at every point $z_{0} \in \mathbf{D}$, see e.g. [MRSY, Theorems 8.1 and 8.6]. It is easy to see that all conditions of Theorem 5.1 are satisfied, except, possibly, the condition $d^{\prime}(f(A)) \geqslant \delta$ in the definition of the class $\mathfrak{F}_{Q, \delta, A}(D)$. We may take here $Q(z) \equiv 1$ and $\delta=1$, because $f$ omit infinitely many continua of diameter greater than 1 outside of the unit disk $\mathbf{D}$.

If, for instance, $\theta=0, a=1 / n, n=1,2, \ldots$, then $f_{n}(z)=\frac{z-1 / n}{1-z / n}=\frac{n z-1}{n-z}$. Let $A=[0,1 / 2]$. Now $f_{n}(0)=-1 / n \rightarrow 0$ and $f_{n}(1 / 2)=\frac{n-2}{2 n-1} \rightarrow 1 / 2$ as $n \rightarrow \infty$. Thus, $f_{n}$ satisfies the condition $h\left(f_{n}(A)\right) \geqslant \delta$ for $\delta=1 / 4$ and sufficiently large $n \in \mathbf{N}$. Thus, $f_{n} \in \mathfrak{F}_{1,1 / 4,[0,1 / 2]}(\mathbf{D})$ for some $n_{0} \in \mathbf{N}$ and each $n \geqslant n_{0}$.

Now, let $g_{n}(z)=\frac{z-(n-1) / n}{1-z(n-1) / n}=\frac{n z-n+1}{n-n z+1}$. It is easy to see, that $g_{n}(z)$ converges locally uniformly to -1 inside of $\mathbf{D}$, but $g_{n}(1)=1$. Thus, $g_{n}$ is not equicontinuous at $z_{0}=1$ and, consequently, $g_{n} \notin \mathfrak{F}_{1,1, A}(D)$ for any continua $A$ in $\mathbf{D}$ by Theorem 5.1 . However, $g_{n} \in \mathfrak{R}_{1,1}(\mathbf{D})$. Thus, $\mathfrak{F}_{Q, \delta, A}(D) \subset \mathfrak{R}_{Q, \delta}(D)$ and $\mathfrak{F}_{Q, \delta, A}(D) \neq \mathfrak{R}_{Q, \delta}(D)$, in general.

Here we took into account that all prime ends of the unit disk are singletons, see $\left[\mathrm{Na}_{4}\right.$, Theorem 4.1].

Remark 5.2. We give one simple example of family ring $Q$-mappings between metric spaces, different from $\mathbf{R}^{n}$. Let $h$ be a hyperbolic metric, and let $d s_{h}$ and $d h$ are elements of hyperbolic length and area, correspondingly, see (1.12) and (1.13). We write $\rho \in \operatorname{adm}_{e} \Gamma$, if $\int_{\gamma} \rho(z)|d z| \geqslant 1$ for every (locally rectifiable) path $\gamma \in \Gamma$. Put $M_{e}(\Gamma):=\inf _{\rho \in \operatorname{adm}_{e} \Gamma} \int_{\mathbf{D}} \rho^{2}(z) d m(z)$. Similarly, $\rho \in \operatorname{adm}_{h} \Gamma$, if $\int_{\gamma} \rho(z) d s_{h}(z) \geqslant 1$ for every (locally rectifiable) path $\gamma \in \Gamma$. Define $M_{h}(\Gamma):=\inf _{\rho \in \operatorname{adm}_{h} \Gamma} \int_{\mathbf{D}} \rho^{2}(z) d h(z)$.

1) Observe that

$$
M_{h}(\Gamma)=M_{e}(\Gamma)
$$

Indeed, if $\rho \in \operatorname{adm}_{e} \Gamma$, then $\int_{\gamma} \rho(z)|d z| \geqslant 1$ for $\gamma \in \Gamma$ and, thus, $\rho_{1}(z):=\frac{\left(1-|z|^{2}\right) \rho(z)}{2} \in$ $\operatorname{adm}_{h} \Gamma$, because $\int_{\gamma} \rho_{1}(z) d s_{h}(z)=\int_{\gamma} 2 \frac{\left(1-|z|^{2}\right) \rho(z)}{2\left(1-|z|^{2}\right)}|d z| \geqslant 1$ for $\gamma \in \Gamma$. Thus, $M_{h}(\Gamma) \leqslant$ $\int_{\mathbf{D}} 4 \frac{\left(1-|z|^{2}\right)^{2} \rho^{2}(z)}{4\left(1-|z|^{2}\right)^{2}} d m(z)=\int_{\mathbf{D}} \rho^{2}(z) d m(z)$. Letting here to inf over $\rho \in \operatorname{adm}_{e} \Gamma$, we obtain that $M_{h}(\Gamma) \leqslant M_{e}(\Gamma)$. The inequality $M_{e}(\Gamma) \leqslant M_{h}(\Gamma)$ can be obtained similarly.

2) Given $0<r_{1}<r_{2}<\infty$ and $0<R_{1}<R_{2}<1$, define $A:=\left\{z \in \mathbf{D}: R_{1}<\right.$ $\left.|z|<R_{2}\right\}, \widetilde{A}:=\left\{z \in \mathbf{D}: r_{1}<h(z, 0)<r_{2}\right\}, S_{i}=\left\{z \in \mathbf{D}:|z|=R_{i}\right\}, \widetilde{S}_{i}=\{z \in$ $\left.\mathbf{D}: h(z, 0)=r_{i}\right\}, i=1,2$. Now, we prove the following statement: if $f: \mathbf{D} \rightarrow \mathbf{D}$ satisfies

$$
M_{e}\left(f\left(\Gamma\left(S_{1}, S_{2}, A\right)\right)\right) \leqslant \int_{R_{1}<|z|<R_{2}} Q(z) \eta^{2}(|z|) d m(z)
$$


for $\eta:\left[R_{1}, R_{2}\right] \rightarrow[0, \infty]$ with $\int_{R_{1}}^{R_{2}} \eta(t) d t \geqslant 1$, then

$$
M_{h}\left(f\left(\Gamma\left(\widetilde{S_{1}}, \widetilde{S_{2}}, \widetilde{A}\right)\right)\right) \leqslant \int_{r_{1}<h(z, 0)<r_{2}} Q(z) \zeta^{2}(h(z, 0)) d h(z)
$$

$\zeta:\left[r_{1}, r_{1}\right] \rightarrow[0, \infty], \int_{r_{1}}^{r_{2}} \zeta(t) d t \geqslant 1$. Indeed, assume that (5.12) holds for every $0<R_{1}<R_{2}<1$. Given $0<r_{1}<r_{2}<\infty$, observe that $\widetilde{A}:=\left\{z \in \mathbf{D}: r_{1}<h(z, 0)<\right.$ $\left.r_{2}\right\}=\left\{z \in \mathbf{D}: \frac{e^{r_{1}-1}}{e^{r_{1}+1}}<|z|<\frac{e^{r_{2}-1}}{e^{r_{2}+1}}\right\}$ and $\widetilde{S}_{i}=\left\{z \in \mathbf{D}:|z|=\frac{e^{r_{i}-1}}{e^{r_{i}+1}}\right\}, i=1,2$. Let $\zeta:\left[r_{1}, r_{1}\right] \rightarrow[0, \infty]$ be a Lebesgue measurable function with $\int_{r_{1}}^{r_{2}} \zeta(t) d t \geqslant 1$. Arguing as in the proof of [RV, Lemma 3.1], putting $\eta(r):=\frac{1}{1-r^{2}} \cdot \zeta\left(\log \frac{1+r}{1-r}\right)$, we observe that $\int_{R_{1}}^{R_{2}} \eta(t) d t=1$, where $R_{1}:=\frac{e^{r_{1}-1}}{e^{r_{1}}+1}$ and $R_{2}:=\frac{e^{r_{2}-1}}{e^{r_{2}+1}}$. Thus, (5.13) directly follows from (5.12) and (5.11).

3) Now, let $\mathbf{D} / G$ be a Riemannian surface of hyperbolic type and $\pi: \mathbf{D} \rightarrow \mathbf{D} / G$ is a universal covering mapping of $\mathbf{D}$ onto $\mathbf{D} / G$ (see item 3 ) of Remark 1.3). Let $p_{0} \in \mathbf{D} / G$ be such that $\pi(0)=p_{0}$, let $D_{0}$ be a Dirichlet polygon for $G$ centered at the origin, and let $\psi=\left(\left.\pi\right|_{D_{0}}\right)^{-1}$. Putting $U_{0}:=\pi\left(D_{0}\right)$, we can find a neighborhood $V_{0} \subset U_{0}$ of $p_{0}$ such that $\widetilde{h}\left(p, p_{0}\right)=h(\psi(p), 0)$, where $\widetilde{h}$ is a metric on $\mathbf{D} / G$ defined by $(1.14)$.

Let $p \geqslant 1$ be a number, such that $2 / p<1$. Put $\alpha \in(0,2 / p)$. We define a sequence of mappings $f_{m}: \mathbf{D} \rightarrow \mathbf{D}$ in the following way:

$$
f_{m}(z)= \begin{cases}\frac{1+|z|^{\alpha}}{2|z|} \cdot z, & 1 / m \leqslant|z|<1 \\ \frac{1+(1 / m)^{\alpha}}{2(1 / m)} \cdot z, & 0<|z|<1 / m\end{cases}
$$

Notice, that $f_{m}$ satisfies (5.12) and, consequently, (5.13) for $Q=\frac{1+|z|^{\alpha}}{\alpha|z|^{\alpha}} \in L^{1}(\mathbf{D})$ at every $z_{0} \in \mathbf{D}$, see $\left[\mathrm{Sev}_{2}\right.$, proof of Theorem 7.1]. Now, mappings

$$
g_{m}(p)= \begin{cases}\frac{1+|\psi(p)|^{\alpha}}{2|\psi(p)|} \cdot \psi(p), & p \in V_{0} \cap \pi(1 / m \leqslant|z|<1), \\ \frac{1+(1 / m)^{\alpha}}{2(1 / m)} \cdot \psi(p), & p \in V_{0} \cap \pi(0<|z|<1 / m)\end{cases}
$$

a ring $Q \circ \psi$-homeomorphisms of $V_{0} \subset \mathbf{D} / G$ into $\mathbf{D}$ at $p_{0}$.

Similarly, if $\mathbf{D} / G_{*}$ is another Riemannian surface, and $\pi_{*}: \mathbf{D} \rightarrow \mathbf{D} / G_{*}$ is a universal covering map of $\mathbf{D}$, we may assume that $p_{0}^{*} \in \mathbf{D} / G_{*}$ is some point with $\pi_{*}(0)=p_{0}^{*}$. Let $D_{0}^{*}$ be a Dirichlet polygon for group $G_{*}$ centered at origin, and let $\psi_{*}=\left(\left.\pi_{*}\right|_{D_{0}^{*}}\right)^{-1}$. Putting $U_{0}^{*}:=\pi_{*}\left(D_{0}^{*}\right)$, observe that there exits a neighborhood $V_{0}^{*} \subset U_{0}^{*}$ of $p_{0}^{*}$ such that $\widetilde{h^{*}}\left(p, p_{0}\right)=h\left(\psi_{*}(p), 0\right)$ for $p \in V_{0}^{*}$. Here $\widetilde{h^{*}}$ is a metric on $\mathbf{D} / G_{*}$, defined similarly to (1.14). Let $k \in \mathbf{N}$ be so large that $g_{m}(p) / k \in \psi_{*}\left(V_{0}^{*}\right)$ for every $p \in U_{0}$. Now, mappings $h_{m}(p):=\pi_{*}\left(g_{m}(p) / k\right)$ are ring $Q \circ \psi$-homeomorphisms at $p_{0}$ of $U_{0}$ into $V_{0}^{*}$.

Remark 5.3. Every homeomorphism $f: D \rightarrow \mathbf{S}^{*}$ of Sobolev class with finite distortion between Riemannian surfaces $\mathbf{S}$ and $\mathbf{S}^{*}$ is a ring $Q$-mapping for each $p_{0} \in$ $\bar{D}$, whenever $Q(p)=K_{f}(p)$ is a maximal dilatation of $f$ at $p \in D$ and $K_{f}(p)$ is locally integrable (see, e.g., [RV, Lemma 3.1]). There are known examples of ring Q-homeomorphisms on Carnot and Heisenberg groups (see, e.g., [BFP, p. 7], [UV, Corollaries 2 and 3], cf. [Ma, Theorem 3.1]). We also point out a result for Riemannian manifolds, see [Af, Lemma 6]. 


\section{Equicontinuity of families of maps with A-condition}

Given $\delta>0, D \subset X$ and a measurable function $Q: D \rightarrow[0, \infty]$, denote $\mathfrak{G}_{Q, \delta, \mathbf{A}}(D)$ the family of all open discrete ring $Q$-maps $f: D \rightarrow D^{\prime} \backslash K_{f}$ in $D$ with A-condition, such that $d^{\prime}\left(K_{f}\right)=\sup _{x, y \in K_{f}} d^{\prime}(x, y) \geqslant \delta$, where $K_{f} \subset D^{\prime}$ is a continuum. The following statement holds.

Lemma 6.1. Let $D$ be a domain in locally path connected and locally compact space $X$ with finite Hausdorff dimension $\alpha \geqslant 2$, and let $D^{\prime}$ be a uniform domain with finite Hausdorff dimension $\alpha^{\prime} \geqslant 2$. Given $x_{0} \in D$, assume that conditions (1.15)-(1.16) hold. Now, $\mathfrak{G}_{Q, \delta, \boldsymbol{A}}(D)$ is equicontinuous at $x_{0}$.

Proof. The idea of a proof is closely related to $\left[\mathrm{Sev}_{2}\right.$, Lemma 2], and similar to Lemma 5.1. Assume the contrary, i.e., assume that $\mathfrak{G}_{Q, \delta, \mathbf{A}}(D)$ is not equicontinuous at $x_{0}$. Now, there exists $x_{k} \in D$ and $f_{k} \in \mathfrak{G}_{Q, \delta, \mathbf{A}}(D)$ such that $x_{k} \rightarrow x_{0}$ as $k \rightarrow \infty$ and

$$
d^{\prime}\left(f_{k}\left(x_{k}\right), f_{k}\left(x_{0}\right)\right) \geqslant \varepsilon_{0}
$$

for some $\varepsilon_{0}$. Since $X$ is locally compact metric space, we can consider that $\overline{B\left(x_{0}, \varepsilon_{0}\right)}$ is a compact set in $X$. Since $X$ is locally compact metric space, we can consider that $\overline{B\left(x_{0}, \varepsilon_{0}\right)}$ is a compact set in $X$. Since $X$ is locally connected by assumption, there is a sequence of balls $B\left(x_{0}, \varepsilon_{k}\right), k=0,1,2, \ldots, \varepsilon_{k} \rightarrow 0$ as $k \rightarrow \infty$, such that $V_{k+1} \subset \overline{B\left(x_{0}, \varepsilon_{k}\right)} \subset V_{k}$, where the $V_{k}$ are continua in $D$. There is no loss of generality in assuming that $x_{k} \in V_{k}$. Now, $x_{0}$ and $x_{k}$ can be joined by a curve $\gamma_{k}$ in the domain $V_{k}$.

By [Sev, Lemma 3], (1.16) implies that

$$
M_{\alpha^{\prime}}\left(\Gamma\left(f_{k}\left(\overline{B\left(x_{0}, \varepsilon\right)}\right), \partial f_{k}\left(B\left(x_{0}, \varepsilon_{0}\right)\right), D^{\prime}\right)\right) \leqslant \alpha(\varepsilon)
$$

as $\varepsilon \rightarrow 0$, where $\alpha(\varepsilon)$ is some function with $\alpha(\varepsilon) \rightarrow 0$ as $\varepsilon \rightarrow 0$. Thus, we obtain from (6.2) that

$$
M_{\alpha^{\prime}}\left(\Gamma\left(f_{k}\left(\gamma_{k}\right), \partial f_{k}\left(B\left(x_{0}, \varepsilon_{0}\right)\right), D^{\prime}\right)\right) \leqslant \alpha\left(\varepsilon_{k-1}\right) \rightarrow 0, \quad k \rightarrow \infty .
$$

On the other hand, observe that $\Gamma\left(K_{f_{k}}, f_{k}\left(\gamma_{k}\right), D^{\prime}\right)>\Gamma\left(f_{k}\left(\gamma_{k}\right), \partial f_{k}\left(B\left(x_{0}, \varepsilon_{0}\right)\right), D^{\prime}\right)$ (see $[\mathrm{Ku}, \mathrm{Ch} .5, \S 46$, item I]); consequently, by (1.2) we obtain

$$
M_{\alpha^{\prime}}\left(\Gamma\left(K_{f_{k}}, f_{k}\left(\gamma_{k}\right), D^{\prime}\right)\right) \leqslant M_{\alpha^{\prime}}\left(\Gamma\left(f_{k}\left(\gamma_{k}\right), \partial f_{k}\left(B\left(x_{0}, \varepsilon_{0}\right)\right), D^{\prime}\right)\right) .
$$

By $(6.1)$, we obtain that $d^{\prime}\left(f_{k}\left(\gamma_{k}\right)\right) \geqslant \varepsilon_{0}$ for every $k=1,2, \ldots$, moreover, $d^{\prime}\left(K_{f_{k}}\right) \geqslant \delta$ for every $k=1,2, \ldots$, by assumption of the lemma. Now, since $D^{\prime}$ is a uniform domain, we obtain that

$$
M_{\alpha^{\prime}}\left(\Gamma\left(K_{f_{k}}, f_{k}\left(\gamma_{k}\right), D^{\prime}\right)\right) \geqslant r_{0}
$$

for each $k=1,2, \ldots$, and some $r_{0}>0$. Observe that (6.5) contradicts with (6.3) and (6.4). Thus, $\mathfrak{G}_{Q, \delta, \mathbf{A}}(D)$ is equicontinuous at $x_{0}$, as required.

Let $\delta>0$, let $D \subset X$ and let $Q: D \rightarrow[0, \infty]$ be a measurable function. Denote $\mathfrak{E}_{Q, \delta, \mathbf{A}}(D)$ the family of all open closed discrete ring $Q$-maps $f: D \rightarrow X^{\prime}$ in $\bar{D}$ with the following conditions: 1) $f$ satisfies A-condition in $D$; 2) given $f: D \rightarrow X^{\prime}$ there exists a continuum $K_{f} \subset X^{\prime} \backslash f(D)$ and $\left.d^{\prime}\left(K_{f}\right)=\sup _{x, y \in K_{f}} d^{\prime}(x, y) \geqslant \delta ; 3\right)$ given $f: D \rightarrow X^{\prime}$ there exists a continuum $A_{f} \subset f(D)$ such that $d^{\prime}\left(A_{f}\right) \geqslant \delta$ and $d\left(f^{-1}\left(A_{f}\right), \partial D\right) \geqslant \delta$. The following statement holds.

Lemma 6.2. Let $D$ and $D_{f}^{\prime}:=f(D), f \in \mathfrak{E}_{Q, \delta, \boldsymbol{A}}(D)$, be domains with finite Hausdorff dimensions $\alpha$ and $\alpha^{\prime} \geqslant 2$ in spaces $(X, d, \mu)$ and $\left(X^{\prime}, d^{\prime}, \mu^{\prime}\right)$, respectively, 
and let $X^{\prime}$ be a domain with finite Hausdorff dimension $\alpha^{\prime} \geqslant 2$. Assume that $X$ is complete and supports an $\alpha$-Poincaré inequality, and that the measure is doubling. Let $D$ be a bounded domain which is finitely connected at the boundary, and let $Q: X \rightarrow(0, \infty)$ be a locally integrable function. Assume that, for every $x_{0} \in \bar{D}$, conditions (1.15)-(1.16) hold. If $D_{f}^{\prime}:=f(D)$ and $X^{\prime}$ are equi-uniform domains over $\mathfrak{E}_{Q, \delta, \boldsymbol{A}}(D)$ and $\overline{D_{f}^{\prime}}$ are compacts in $X^{\prime}$, then every $f \in \mathfrak{E}_{Q, \delta, \boldsymbol{A}}(D)$ has a continuous extension $f: \bar{D}_{P} \rightarrow \overline{D_{f}^{\prime}}$, and $\mathfrak{E}_{Q, \delta, \boldsymbol{A}}(D)$ is equicontinuous in $\bar{D}_{P}$.

Proof. Arguing as in the proof of Lemma 5.2, we obtain that $\partial D_{f}^{\prime}=\partial f(D)$ is strongly accessible for every $f \in \mathfrak{E}_{Q, \delta, \mathbf{A}}(D)$. Moreover, we see that $X$ is a locally compact and locally path connected space. Now, by Lemma 2.1 every $f \in \mathfrak{F}_{Q, \delta, A}(D)$ has a continuous extension $f: \bar{D}_{P} \rightarrow \overline{D_{f}^{\prime}}$. By Lemma 6.1 , we also obtain that $\mathfrak{E}_{Q, \delta, \mathbf{A}}(D)$ is equicontinuous in $D$, because $\mathfrak{E}_{Q, \delta, \mathbf{A}}(D) \subset \mathfrak{G}_{Q, \delta, \mathbf{A}}(D)$.

It remains to show that $\mathfrak{E}_{Q, \delta, \mathbf{A}}(D)$ is equicontinuous on $E_{D}:=\bar{D}_{P} \backslash D$. Assume the contrary, i.e., assume that there exists $P_{0} \in E_{D}$ such that $\mathfrak{E}_{Q, \delta, \mathbf{A}}(D)$ is not equicontinuous at $P_{0}$. Now, there is exists $P_{k} \in \bar{D}_{P}$ and $f_{k} \in \mathfrak{E}_{Q, \delta, \mathbf{A}}(D)$ such that $P_{k} \rightarrow P_{0}$ as $k \rightarrow \infty$ and

$$
d^{\prime}\left(f_{k}\left(P_{k}\right), f_{k}\left(P_{0}\right)\right) \geqslant \varepsilon_{0}
$$

for some $\varepsilon_{0}$. Since $f_{k}$ has a continuous extension on $\bar{D}_{P}$, given $k \in \mathbf{N}$, we can find $x_{k} \in D$ with $m_{P}\left(x_{k}, P_{k}\right)<1 / k$ and $d\left(f_{k}\left(x_{k}\right), f_{k}\left(P_{k}\right)\right)<1 / k$. Thus, we obtain from (6.6) that

$$
d^{\prime}\left(f_{k}\left(x_{k}\right), f_{k}\left(P_{0}\right)\right) \geqslant \varepsilon_{0} / 2 \quad \forall k=1,2, \ldots
$$

Similarly, we can find $x_{k}^{\prime} \in D$ such that $x_{k}^{\prime} \rightarrow P_{0}$ as $k \rightarrow \infty$, and $d^{\prime}\left(f_{k}\left(x_{k}^{\prime}\right), f_{k}\left(P_{0}\right)\right)<$ $1 / k, k=1,2, \ldots$ Thus, we obtain from (6.7) that

$$
d^{\prime}\left(f_{k}\left(x_{k}\right), f_{k}\left(x_{k}^{\prime}\right)\right) \geqslant \varepsilon_{0} / 4 \quad \forall k=1,2, \ldots,
$$

where $x_{k}$ and $x_{k}^{\prime} \in D$ satisfy conditions $x_{k} \rightarrow P_{0}, x_{k}^{\prime} \rightarrow P_{0}$ as $k \rightarrow \infty$.

Denote $x_{0}:=I\left(\left[E_{k}\right]\right.$ ) (see Proposition 1.1). By Remark 4.5 in [ABBS] we can consider that the sets $E_{k}$ are open. Moreover, by Remark 2.6 in [ABBS] the set $E_{k}$ is path connected for every $k \in \mathbf{N}$. Arguing as in the proof of Lemma 2.1, we can show that, for every $r>0$ there exists $k \in \mathbf{N}$ such that $E_{k} \subset B\left(x_{0}, r\right) \cap D$. Thus, there is no loss of generality in assuming that $x_{k}, x_{k}^{\prime} \in E_{k}$ and $E_{k} \subset B\left(x_{0}, 2^{-k}\right)$. Let $\gamma_{k}$ be a path, joining $x_{k}$ and $x_{k}^{\prime}$ in $E_{k}$. Let $A_{f_{k}}$ be the set from the definition of $\mathfrak{E}_{Q, \delta, \mathbf{A}}(D)$. Observe that $f_{k}^{-1}\left(A_{f_{k}}\right) \subset D \backslash B\left(x_{0}, 2^{-k}\right)$ for all $k>k_{0}$ and some $k_{0} \in \mathbf{N}$. We can consider that $2^{-k_{0}}<\varepsilon_{0}$. Let $\Gamma_{k}$ be a family of curves joining $\gamma_{k}$ and $f_{k}^{-1}\left(A_{f_{k}}\right)$ in $D$. By Remark 1.1, we obtain that

$$
M_{\alpha^{\prime}}\left(f_{k}\left(\Gamma_{k}\right)\right) \leqslant M_{\alpha^{\prime}}\left(f_{k}\left(\Gamma\left(S\left(x_{0}, 2^{-k}\right), S\left(x_{0}, 2^{-k_{0}}\right), A\left(x_{0}, 2^{-k}, 2^{-k_{0}}\right)\right)\right)\right) .
$$

Observe that

$$
\eta(t)= \begin{cases}\psi(t) / I\left(2^{-k}, 2^{-k_{0}}\right), & t \in\left(2^{-k}, 2^{-k_{0}}\right), \\ 0, & t \in \mathbf{R} \backslash\left(2^{-k}, 2^{-k_{0}}\right),\end{cases}
$$

$I\left(\varepsilon, \varepsilon_{0}\right):=\int_{\varepsilon}^{\varepsilon_{0}} \psi(t) d t$, satisfies the condition (1.6) at $r_{1}=2^{-k}$ and $r_{2}=2^{-k_{0}}$. By the definition of a ring $Q$-homeomorphism at a boundary point, (1.16) and (6.8) imply

$$
M_{\alpha^{\prime}}\left(f_{k}\left(\Gamma_{k}\right)\right) \leqslant \alpha\left(2^{-k}\right) \rightarrow 0
$$

as $k \rightarrow \infty$, where $\alpha(\varepsilon)$ is some nonnegative function with $\alpha(\varepsilon) \rightarrow 0$ as $\varepsilon \rightarrow 0$. 
On the other hand, let us consider the family $\Gamma\left(f_{k}\left(\gamma_{k}\right), A_{f_{k}}, D_{f_{k}}^{\prime}\right)$. Since $D_{f_{k}}^{\prime}:=$ $f_{k}(D)$ are equi-uniform domains, we obtain that

$$
M_{\alpha^{\prime}}\left(\Gamma\left(f_{k}\left(\gamma_{k}\right), A_{f_{k}}, D_{f_{k}}^{\prime}\right)\right) \geqslant r_{0}, \quad k=1,2, \ldots,
$$

for some $r_{0}>0$. Let $\Gamma_{k}^{*}$ be the family of all maximal $f_{k}$-liftings of $\Gamma\left(f_{k}\left(\gamma_{k}\right), A_{f_{k}}, D_{f_{k}}^{\prime}\right)$ starting at $\gamma_{k}$. (The family $\Gamma_{k}^{*}$ is well defined in view of condition A). Arguing as in the proof of Lemma 2.1, we can show that $\Gamma_{k}^{*} \subset \Gamma_{k}$. Besides that, $f_{k}\left(\Gamma_{k}^{*}\right)<$ $\Gamma\left(f_{k}\left(\gamma_{k}\right), A_{f_{k}}, D_{f_{k}}^{\prime}\right)$. Thus, we obtain

$$
M_{\alpha^{\prime}}\left(\Gamma\left(f_{k}\left(\gamma_{k}\right), A_{f_{k}}, D_{f_{k}}^{\prime}\right)\right) \leqslant M_{\alpha^{\prime}}\left(f_{k}\left(\Gamma_{k}^{*}\right)\right) \leqslant M_{\alpha^{\prime}}\left(f_{k}\left(\Gamma_{k}\right)\right) .
$$

But (6.10) and (6.11) contradict with (6.9). Thus, $\mathfrak{E}_{Q, \delta, \mathbf{A}}(D)$ is equicontinuous at $P_{0}$, as required.

The following main result holds.

Theorem 6.1. Let $D$ and $D_{f}^{\prime}:=f(D), f \in \mathfrak{E}_{Q, \delta, \boldsymbol{A}}(D)$, be domains with finite Hausdorff dimensions $\alpha$ and $\alpha^{\prime} \geqslant 2$ in spaces $(X, d, \mu)$ and $\left(X^{\prime}, d^{\prime}, \mu^{\prime}\right)$, respectively, and let $X^{\prime}$ be a domain with finite Hausdorff dimension $\alpha^{\prime} \geqslant 2$. Assume that $X$ is complete and supports an $\alpha$-Poincaré inequality, and that the measure is doubling. Let $D$ be a bounded domain which is finitely connected at the boundary, and let $Q: X \rightarrow(0, \infty)$ be a locally integrable function. Assume that $Q \in F M O(\bar{D})$. If $D_{f}^{\prime}:=f(D)$ and $X^{\prime}$ are equi-uniform domains over $f \in \mathfrak{E}_{Q, \delta, \boldsymbol{A}}(D)$ and $\overline{D_{f}^{\prime}}$ are compact in $X^{\prime}$, then every $f \in \mathfrak{E}_{Q, \delta, \boldsymbol{A}}(D)$ has a continuous extension $f: \bar{D}_{P} \rightarrow \overline{D_{f}^{\prime}}$, and $\mathfrak{E}_{Q, \delta, \boldsymbol{A}}(D)$ is equicontinuous in $\bar{D}_{P}$.

Proof of the Theorem 6.1 follows from Lemma 6.2 and Proposition 3.1. Indeed, $X$ is upper regular by (1.1), and (3.1) holds because the measure is doubling by assumptions. So, the desired statement follows from the Lemma 6.2.

Acknowledgments. Author thanks Professor Tomasz Adamowicz, Institute of Mathematics of Polish Academy of Science, Warsaw, for joint discussion and useful recommendations.

\section{References}

[A] Adamowicz, T.: Prime ends in metric spaces and boundary extensions of mappings. arXiv:1608.02393.

[ABBS] Adamowicz, T., A. BJörn, J. BJörn, and N. Shanmugalingam: Prime ends for domains in metric spaces. - Adv. Math. 238, 2013, 459-505.

[Af] Afanas'Eva, E. S.: Boundary behavior of ring $Q$-homeomorphisms on Riemannian manifolds. - Ukrainian Math. J. 63:10, 2012, 1479-1493.

[BFP] Balogh, Z. M., K. Fässler, and K. Peltonen: Uniformly quasiregular maps on the compactified Heisenberg group. - J. Geom. Anal. 22:3, 2012, 633-665.

[Berd] Beardon, A. F.: The geometry of discrete groups. - Grad. Texts in Math. 91, SpringerVerlag, New York, 1983.

[BB] BJöRN, A., and J. BJÖRN: Nonlinear potential theory on metric spaces. - In: EMS Tracts in Math. 17, Eur. Math. Soc., Zurich, 2011.

[Ch] CheEger, J.: Differentiability of Lipschitz functions on metric measure spaces. - Geom. Funct. Anal. 9, 1999, 428-517.

[ES] Estep, D., and N. Shanmugalingam:' Geometry of prime end boundary and the Dirichlet problem for bounded domains in metric measure spaces. - Potential Anal. 42:2, 2015, $335-363$. 
[GRY] GutlyanskiI, V. Ya., V. I. Ryazanov, and E. Yakubov: The Beltrami equations and prime ends. - Ukr. Mat. Visn. 12:1, 2015, 27-66; English transl. in J. Math. Sci. (N.Y.) $210: 1,2015,22-51$.

[HR] Heinonen, J., and S. Rickman: Geometric branched covers between generalized manifolds. - Duke Math. J. 113, 2002, 465-529.

[Fu] Fuglede, B.: Extremal length and functional completion. - Acta Math. 98, 1957, 171-219.

[IR] Ignat'Ev, A., and V. Ryazanov: Finite mean oscillation in mapping theory. - Ukr. Mat. Visn. 2:3, 2005, 395-417 (in Russian); English transl. in Ukr. Math. Bull. 2:3, 2005, 403-424.

[IS] IL'yutKo, D. P., and E. A. Sevost'yanov: Open discrete mappings with unbounded coefficient of quasiconformality on Riemannian manifolds. - Mat. Sb. 207:4, 2016, 65-112 (in Russian); English transl. in Sb. Math. 207:4, 2016, 537-580.

[KR] Kovtonyuk, D. A., and V. I. Ryazanov: On the theory of prime ends for space mappings. - Ukr. Mat. Zh. 67:4, 2015, 467-479; English transl. in Ukrainian Math. J. 67:4, $2015,528-541$.

[Ku] Kuratowski, K.: Topology, vol. 2. - Academic Press, New York-London, 1968.

[Ma] Markina, I.: On coincidence of $p$-module of a family of curves and $p$-capacity on the Carnot group. - Rev. Mat. Iberoamericana 19, 2003, 143-160.

[MV] Markina, I., and S. Vodopyanov: On value distributions for quasimeromorphic mappings on H-type Carnot groups. - Bull. Sci. Math. 130, 2006, 467-523.

[MRV] Martio, O., S. Rickman, and J. VÄISÄLÄ: Topological and metric properties of quasiregular mappings. - Ann. Acad. Sci. Fenn. Ser. A I Math. 488, 1971, 1-31.

[MRSY] Martio, O., V. Ryazanov, U. Srebro, and E. Yakubov: Moduli in modern mapping theory. - Springer Science + Business Media, LLC, New York, 2009.

[MS] Martio, O., and J. Sarvas: Injectivity theorems in plane and space. - Ann. Acad. Sci. Fenn. Ser. A I Math. 4, 1978/1979, 384-401.

[Na $\mathrm{Na}_{1}$ NÄKKI, R.: Boundary behavior of quasiconformal mappings in $n$-space. - Ann. Acad. Sci. Fenn. Ser. A I Math. 484, 1970, 1-50.

[Na $\mathrm{Na}_{2}$ NÄKKI, R.: Continuous boundary extension of quasiconformal mappings. - Ann. Acad. Sci. Fenn. Ser. A I Math. 511, 1972, 1-10.

[Na $\mathrm{Na}_{3}$ NÄKKI, R.: Extension of Loewner's capacity theorem. - Trans. Amer. Math. Soc. 180, 1973, 229-236.

[Na $\mathrm{Na}_{4}$ NÄKKI, R.: Prime ends and quasiconformal mappings. - J. Anal. Math. 35, 1979, 13-40.

[NP] NÄKKI, R., and B. PALKA: Uniform equicontinuity of quasiconformal mappings. - Proc. Amer. Math. Soc. 37:2, 1973, 427-433.

[OR] Onninen, J., and K. Rajala: Quasiregular maps to generalized manifolds. - J. Anal. Math. 109, 2009, 33-79.

[RR] Reimann, H. M., and T. Rychener: Funktionen Beschränkter Mittlerer Oscillation. Lecture Notes in Math. 487, Springer-Verlag, Berlin etc., 1975.

[Ri] Rickman, S.: Quasiregular mappings. - Springer-Verlag, Berlin etc., 1993.

[RS] Ryazanov, V., and R. Salimov: Weakly planar spaces and boundaries in the theory of mappings. - Ukr. Mat. Visn. 4:2, 2007, 199-234 (in Russian); English transl. in Ukr. Math. Bull. 4:2, 2007, 199-234.

[RV] RYazAnov, V., and S. Volkov: On the boundary behavior of mappings in the class $W_{\text {loc }}^{1,1}$ on Riemann surfaces. - Complex Anal. Oper, Theory 11, 2017, 1503-1520.

[Se] Semmes, S.: Some novel types of fractal geometry. - Clarendon Press, Oxford, 2001.

[Sev 1 Sevost'Yanov, E.: On boundary behavior of mappings in terms of prime ends. arXiv:1602.00660. 
[Sev 2 Sevost'Yanov, E.: Local and boundary behavior of maps in metric spaces. - Algebra i analiz, 28:6, 2016, 118-146 (in Russian); English transl. in St. Petersburg Math. J. 28:6, 2017, 807-824.

[Sm] SmolovayA, E. S.: Boundary behavior of ring $Q$-homeomorphisms in metric spaces. Ukrainian Math. J. 62:5, 2010, 785-793.

[UV] Ukhlov, A., and S.K. Vodop'Yanov: Mappings with bounded $(P, Q)$-distortion on Carnot groups. - Bull. Sci. Math. 134, 2010, 605-634.

[Va] VÄIsÄLÄ, J.: Lectures on $n$-dimensional quasiconformal mappings. - Lecture Notes in Math. 229, Springer-Verlag, Berlin etc., 1971.

$\left[\mathrm{Vu}_{1}\right]$ VuORINEN, M.: Exceptional sets and boundary behavior of quasiregular mappings in $n$-space. - Ann. Acad. Sci. Fenn. Ser. A I Math. 11, 1976, 1-44.

[ $\left.\mathrm{Vu}_{2}\right] \quad$ VuORINEN, M.: On the existence of angular limits of $n$-dimensional quasiconformal mappings. - Ark. Math. 18, 1980, 157-180.

Received 3 January 2018 • Revised received 8 June 2018 • Accepted 24 July 2018 